\title{
Evaluation of Aromatic Plants and Compounds Used to Fight Multidrug Resistant Infections
}

\author{
Ramar Perumal Samy, ${ }^{1}$ Jayapal Manikandan, ${ }^{2,3}$ and Mohammed Al Qahtani ${ }^{2}$ \\ ${ }^{1}$ Infectious Diseases Programme, MD4, 5 Science Drive 2, Department of Microbiology, Yong Loo Lin School of Medicine, \\ National University Health System (NUHS), National University of Singapore, Singapore 117597 \\ ${ }^{2}$ Center of Excellence in Genomic Medicine Research, King Abdulaziz University, P.O. Box 80216, Jeddah 21589, Saudi Arabia \\ ${ }^{3}$ School of Anatomy, Physiology and Human Biology, The University of Western Australia, 35 Stirling Highway, \\ Crawley, WA 6009, Australia
}

Correspondence should be addressed to Ramar Perumal Samy; rperumalsamy@yahoo.co.uk and Mohammed Al Qahtani; mhalqahtani@kau.edu.sa

Received 29 November 2012; Revised 7 May 2013; Accepted 23 May 2013

Academic Editor: Rong Zeng

Copyright (C) 2013 Ramar Perumal Samy et al. This is an open access article distributed under the Creative Commons Attribution License, which permits unrestricted use, distribution, and reproduction in any medium, provided the original work is properly cited.

\begin{abstract}
Traditional medicine plays a vital role for primary health care in India, where it is widely practiced to treat various ailments. Among those obtained from the healers, 78 medicinal plants were scientifically evaluated for antibacterial activity. Methanol extract of plants (100 $\mu \mathrm{g}$ of residue) was tested against the multidrug resistant (MDR) Gram-negative and Gram-positive bacteria. Fortyseven plants showed strong activity against Burkholderia pseudomallei (strain TES and KHW) and Staphylococcus aureus, of which Tragia involucrata L., Citrus acida Roxb. Hook.f., and Aegle marmelos (L.) Correa ex Roxb. showed powerful inhibition of bacteria. Eighteen plants displayed only a moderate effect, while six plants failed to provide any evidence of inhibition against the tested bacteria. Purified compounds showed higher antimicrobial activity than crude extracts. The compounds showed less toxic effect to the human skin fibroblasts (HEPK) cells than their corresponding aromatic fractions. Phytochemical screening indicates that the presence of various secondary metabolites may be responsible for this activity. Most of the plant extracts contained high levels of phenolic or polyphenolic compounds and exhibited activity against MDR pathogens. In conclusion, plants are promising agents that deserve further exploration. Lead molecules available from such extracts may serve as potential antimicrobial agents for future drug development to combat diseases caused by the MDR bacterial strains as reported in this study.
\end{abstract}

\section{Introduction}

Treatment of infections is compromised worldwide by the emergence of bacteria that are resistant to multiple antibiotics [1]. New and emerging drug resistance bacteria strains, particularly methicillin-resistant Staphylococcus aureus (MRSA), vancomycin-resistant enterococci (VRE), Mycobacterium tuberculosis (MTB), and multidrug resistance (MDR) Gramnegative bacteria, are increasing worldwide and add to the gravity of the situation [2]. S. aureus cause a variety of syndromes such as food poisoning, toxic shock syndrome, skin lesions [3], hyperproliferative skin disease [4], and atopic dermatitis [5, 6]. Community-acquired pneumonia caused by Streptococcus pneumoniae, Klebsiella pneumonia, and S. aureus accounts for significant mortality in Southeast Asia [7]. Melioidosis has been recognized as an important human infection caused by Burkholderia pseudomallei in Singapore, Malaysia, Thailand, and Northern Australia $[8,9]$. Cases have also been reported from some other tropical and subtropical regions like Africa and America, and a number of cases in man has recently been reported to increase in China, Taiwan, and South India $[10,11]$. Infection with antibiotic resistant bacteria negatively impacts on public health, due to an increased incidence of treatment failure and severity of diseases. Development of resistant bacteria due to the chromosomal mutations is more commonly associated with the horizontal transfer of resistance determinants borne on mobile genetic elements [12]. B. pseudomallei is intrinsically 
resistant to many antibiotics $[13,14]$. Considering the higher cost for producing synthetic drugs and the various side effects associated with their use, the need to search for alternative agents from medicinal plants and essential oils used in folklore medicine is further justified to overcome these issues. In India, there are about 550 tribal communities covered under 227 ethnic groups residing in about 5000 villages throughout different forest and vegetation regions [15]. India is one of the world's 12 megabiodiversity countries $[16,17]$. Plant derived medicines have played a major role in human societies throughout the history and prehistory of mankind [18]. The traditional healers (traditional physicians) or medicinemen have a long history of their own diagnostic and treatment system, which they have acquired from their ancestors [19]. Approximately $80 \%$ of the world population still relies on traditional medicine for the treatment of common diseases [20-22]. Medicinal plants thus offer significant potential for the development of novel antibacterial therapies and adjunct treatments [23]. Plant derived drugs serve as a prototype to develop more effective and less toxic medicines. In previous studies, few attempts were made to confirm the antimicrobial activity of some indigenous medicinal plants $[24,25]$. Not only extracts of various medicinal plants but also essential oils and their constituents have been investigated for their antimicrobial properties against bacteria and fungi [26-28].

The principal compounds from the leaves showed a better antibacterial activity against $P$. aeruginosa and $B$. subtilis bacteria and a significant antifungal activity on $C$. albicans [29]. The essential oil from $R$. officinalis (alpha pinene/ verbenone/bornyl acetate) was found to be more sensitive to the Gram-positive bacteria (MIC 2.5-4 mg/mL) than to the Gram-negative bacteria [30]. Several essential oils kill bacteria by damaging the cell membrane structure and inhibiting their membrane function [31]. Because of the antimicrobial potency of plant extracts and oils, they become a rich source of raw materials for many biotechnological and pharmaceutical industries for the development of therapeutic drugs. The increasing trend in the use of aromatic plants and essential oils in food, cosmetic, and pharmaceutical industries suggests that a systematic study of traditional medicinal plants is very important in order to find active compounds from such sources [32-34]. The purpose of this study is to survey and investigate popular medicinal aromatic plants and their essential oils with a view to fight against multidrug resistant human pathogens. In the present study, 71 plant species were selected on the basis of the available medicinal information and screened for their in vitro antimicrobial efficacy against bacteria.

\section{Materials and Methods}

2.1. Ethnomedicinal Survey and Collection of Plants. Ethnomedicinal surveys were conducted during March 1998 and July 2001 from various tribal localities (Kolli hills, Kalrayan hills, Pachamalai, Javadi hills, Mundanthurai) of Eastern and Western Ghats, Tamil Nadu, India. For ethnobotanical studies, questioners were used to collect the general information on the tribes, and the key information on medicinal details was collected through interviews. The medicinal plants were identified by a taxonomist using the standard Flora of Tamil Nadu Carnatic [35], and the voucher specimens were deposited in the department's herbarium at the Entomology Research Institute, Loyola College, Chennai, India.

2.2. Preparation of Plant Extracts. Using a Soxhlet apparatus, the shade-dried and powdered plant materials (200 g of each) were extracted with $1000 \mathrm{~mL}$ of methanol $\left(\mathrm{CH}_{3} \mathrm{OH}\right)$ for $10 \mathrm{~h}$. The collected methanol extracts were filtered (Whatman no. 1 filter paper) and evaporated with a rotary evaporator and freeze dryer (lyophilized) to obtain the crude extracts (Buchi, Labortechnik AG, Switzerland). The dried crude extracts were stored at $4^{\circ} \mathrm{C}$ for antimicrobial assays [34].

2.3. Culture of Microorganisms. The following Gram-negative: Burkholderia pseudomallei (TES21), Burkholderia pseudomallei (KHW22), Klebsiella pneumoniae (ATCC15380), Klebsiella pneumoniae, Pseudomonas aeruginosa (ATCC27853), Vibrio damsela, and Salmonella typhi (ATCC51812) and Gram-positive: Staphylococcus aureus (ATCC 29213), Streptococcus pyogenes, and Streptococcus pneumoniae (ATCC49619) microorganisms were used for cultures. B. pseudomallei bacterial strains such as KHW and TES were isolated from the patient samples obtained from the Department of Microbiology, NUS, Singapore. The strains were subcultured on $20 \mathrm{~mL}$ Tryptic Soy (TS) and Mueller Hinton (MH) agar plates ( $\mathrm{pH}$ 7.4) and incubated overnight at $37^{\circ} \mathrm{C}$ before use.

2.4. Antimicrobial Activity. The standard bacterial cultures were stored at $-70^{\circ} \mathrm{C}$, subcultured on $20 \mathrm{~mL} \mathrm{MH}$ and TS agar plates ( $\mathrm{pH} 7.4$ ), and incubated overnight at $37^{\circ} \mathrm{C}$ prior to use. The antimicrobial property was tested using the discdiffusion method [36]. Five young colonies of each strain of bacteria taken from their respective cultures grown overnight on TS agar plates (Oxoid limited, Wode Road, Basingstoke, Hants, England, UK) were suspended in $5 \mathrm{~mL}$ of sterile saline $(0.9 \%)$, and the density of the suspension was adjusted to approximately $3 \times 10^{8}$ colony forming unit (CFU). The swab was used to inoculate the dried surface of TS agar plate by streaking four times over the surface of the agar and rotating the plate approximately $90^{\circ} \mathrm{C}$ to ensure an even distribution of the inoculums. The medium was allowed to dry for about $3 \mathrm{~min}$ before adding a 6 millimeter in diameter ( $\mathrm{mm})$ sterile paper disc (Becton Dickinson, USA) on the surface. Each disc was tapped gently down onto the agar to provide a uniform contact. Lyophilized residue $(100 \mu \mathrm{g} / \mathrm{mL})$ of each plant extracts and purified fractions was weighed and dissolved in $1 \mathrm{~mL}$ of water, and $20 \mu \mathrm{L}$ of the extracts and oils (containing $100 \mu \mathrm{g}$ of residue) were applied on each disc (3 replicates), while the sterile blank disc served as a normal control. The antimicrobial effect of the extracts on the clinical isolates was determined in comparison with the reference antibiotics (chloramphenicol $30 \mu \mathrm{g} / \mathrm{disc}$ and ceftazidime $30 \mu \mathrm{g} / \mathrm{disc}$ ), which were used as positive controls. The plates were incubated at $37^{\circ} \mathrm{C}$ for $24 \mathrm{~h}$, and the inhibition zones were measured and calculated. 
2.5. Minimum Inhibitory Concentrations (MICs) Assay. MICs were evaluated based on the in vitro screening of 16 purified fractions that were found to have potent antimicrobial activity. Broth dilution method was used for the MIC assay with some slight modifications as recommend by the NCCLS [37]. Two-fold serial dilutions of all the fractionated compounds were made with $\mathrm{MH}$ and TS broth in microtiter plate wells to adjust the final concentration from 7.8 to $250 \mu \mathrm{g} / \mathrm{mL}$, while wells containing the broth alone without any sample served as a control. Three replicates $(n=3)$ were used for each dilution and culture containing approximately $1 \times 10^{5} \mathrm{CFU} / \mathrm{mL}$. The plates were incubated at $37^{\circ} \mathrm{C}$ for $24 \mathrm{~h}$, and the absorbance was measured at $560 \mathrm{~nm}$.

2.6. Cytotoxicity Assay. The cytotoxic effects of various extracts were tested by MTT assay [38] using human skin fibroblast HEPK cells. The toxic effect of plant extracts and essential oils was assayed on human skin fibroblast (HEPK) cell proliferation in 96-well microtitre plates. Confluent cells $\left(5 \times 10^{6}\right.$ cells per well $)$ were incubated with extracts and oils for $24 \mathrm{~h}$, and the percentage inhibitory concentration $\left(\mathrm{IC}_{50}\right)$ was determined.

2.7. Phytochemical Analysis of Plant Extracts. The most active extracts were used for purification of antimicrobial compounds [39]. $100 \mathrm{~g}$ each of the plant powder was percolated with $500 \mathrm{~mL}$ of $4 \%$ aqueous $\mathrm{HCl}$ (adjusted to $\mathrm{pH} 2$ ) and heated at $50^{\circ} \mathrm{C}$ for $3 \mathrm{~h}$. The extract was washed with $2 \times 250 \mathrm{~mL}$ of diethyl ether, and the organic phase evaporated to dryness using vacuum rotary evaporator. The dark brown gummy residues obtained by acid hydrolysis were chromatographed on silica gel column $60 \times 3.2 \mathrm{~cm}(60-120$ mesh, $\mathrm{pH} 7$, Merck) by gradually eluting with $n$-hexane/ethyl acetate $(8: 2 ; 6: 4$; $3: 7$ and $1: 1)$ and chloroform/methanol $(3: 2)$. The aliquots of each fraction were subjected to thin layer chromatography (TLC) on silica gel coated TLC plate ( $1 \mathrm{~mm}$ Merck) using the solvent system consisting of $20 \%(\mathrm{v} / \mathrm{v})$ n-hexane/ethyl acetate. The chromatograms were detected using $50 \% \mathrm{H}_{2} \mathrm{SO}_{4}$ solution as a spray reagent [34]. The individual fractions were collected and concentrated by vacuum rotary evaporator at $40^{\circ} \mathrm{C}$. All the purified compounds recovered from the silica gel column were monitored by reading the absorbance at 190-350 nm (UV spectrophotometer, Hitachi, Japan). The active fractions were further purified, and the final yields of the compounds were recorded. The lyophilized pooled concentrated compounds were then assayed $(100 \mu \mathrm{g} / \mathrm{mL})$ against bacteria. The phytochemical screening was done on the pure compounds using the chemical method previously reported for the detection of secondary metabolites [40]. The different chemical constituents tested include alkaloids, flavonoids, glycosides, polyphenols, saponin, sterols, triterpenes, tannins, reducing sugars, gallic acid, catechol, and aglycones.

2.8. Statistical Analysis. The bacterial growth inhibitory activity (inhibition zones millimeter in diameter) was compared for significant differences within the bacterial strains. One way analysis of variance was performed (mean $\pm S D$, $n=3$ replicates) using GraphPad Prism 4 , USA. ${ }^{*} P<0.01$ was considered statistically significant (inhibition zones of extracts/fractions versus antibiotic drugs).

\section{Results}

3.1. Study Area for the Collection of Aromatic Medicinal Plants. The Western and Eastern Ghats were selected for the present study with the cite map showing the landmarks (Figures 1(a)1(b)). Kalrayan hills are situated north of Attur taluk (Salem district), one of the major range of hills in the Eastern Ghats of Tamil Nadu (Figure 1(c)). Pachamalai hills are situated to the north of Thuraiyur taluk of Tiruchirappalli district. The rich biodiversity part of Eastern Ghats lies between latitudes $11^{\circ} 09^{\prime} 00^{\prime \prime}$ to $11^{\circ} 27^{\prime} 00^{\prime \prime} \mathrm{N}$ and longitudes $78^{\circ} 28^{\prime} 00^{\prime \prime}$ to $78^{\circ} 49^{\prime} 00^{\prime \prime} \mathrm{E}$, and occupies an area of about 527.61 square $\mathrm{km}$. It is located near $11^{\circ} 11^{\prime} \mathrm{N} 78^{\circ} 21 \mathrm{E} / 11.18^{\circ} \mathrm{N} 78.35^{\circ} \mathrm{E} / 11.18$; 78.35 (Figure $1(\mathrm{~d})$ ). Mundanthurai is located nearly $45 \mathrm{~km}$ west of Tirunelveli district, TN, between latitude $8^{\circ} 25^{\prime}$ and $8^{\circ} 53^{\prime} \mathrm{N}$ and longitude $77^{\circ} 10^{\prime}$ and $77^{\circ} 35^{\prime} \mathrm{E}$. This is the only area of Western Ghats that has the longest raining period of about 8 months and forms the catchment area for 14 rivers and streams (Figure 1(e)). Kolli Malai is a small mountain range located in Namakkal district. The mountains are about $1000-1300 \mathrm{~m}$ in height and cover an area of approximately $280 \mathrm{~km}$. The Kolli hills are part of the Eastern Ghats, which is a mountain range that runs mostly parallel to the east coast of Tamil Nadu in South India (Figure 1(f)). Javadi hills are one of the largest in the Eastern Ghats in Vellore district in the northern part of the state of Tamil Nadu. They consist of bluish gray hills, with peaks averaging 3600-3800 feet or 1100-1150 meter (Figure $1(\mathrm{~g})$ ). Based on the vegetation type (Figures 2(a)-2(d)), the study area consists of (i) dry, deciduous, (ii) moist deciduous, and (iii) rain forests and diverse proportion of plant parts in abundance (Figures 2(e)2(f)). Three different types of tribes (i.e., Kani, Malayali and Paliyan tribes) inhabit in the hill ranges. The Kani tribes, located at Mundanthurai, raise different types of vegetables in their own fields, while the Malayali tribes cultivate rice. They all engage not only in the agricultural work but also are involved in silvicultural work assigned by the forest department, Government of TN, India.

3.2. Medicinal Plants Glory. Western Ghats (Mundanthurai) and Eastern Ghats (Kolli hills, Javadi hills, Kalrayan hills, Pachamalai hills) possess a rich diversity of medicinal plants that are used as food and drug by different groups of tribal communities. Urbanization, habitat degradation, and fragmentation of these forests have resulted in the depletion of natural resources on which these tribes used to depend for their livelihoods. It has become increasingly difficult for them to live in their traditional way. In addition, the impact of modernization and urbanization has encroached in and around tribal settlements, thus changing their lifestyles.

3.3. Plants Valued as Edibles. Various types of plant parts are collected during different seasons, cooked, and eaten along with boiled rice (Table 1). For example, Solanum nigrum leaf is most commonly used in all the four regions. There are 


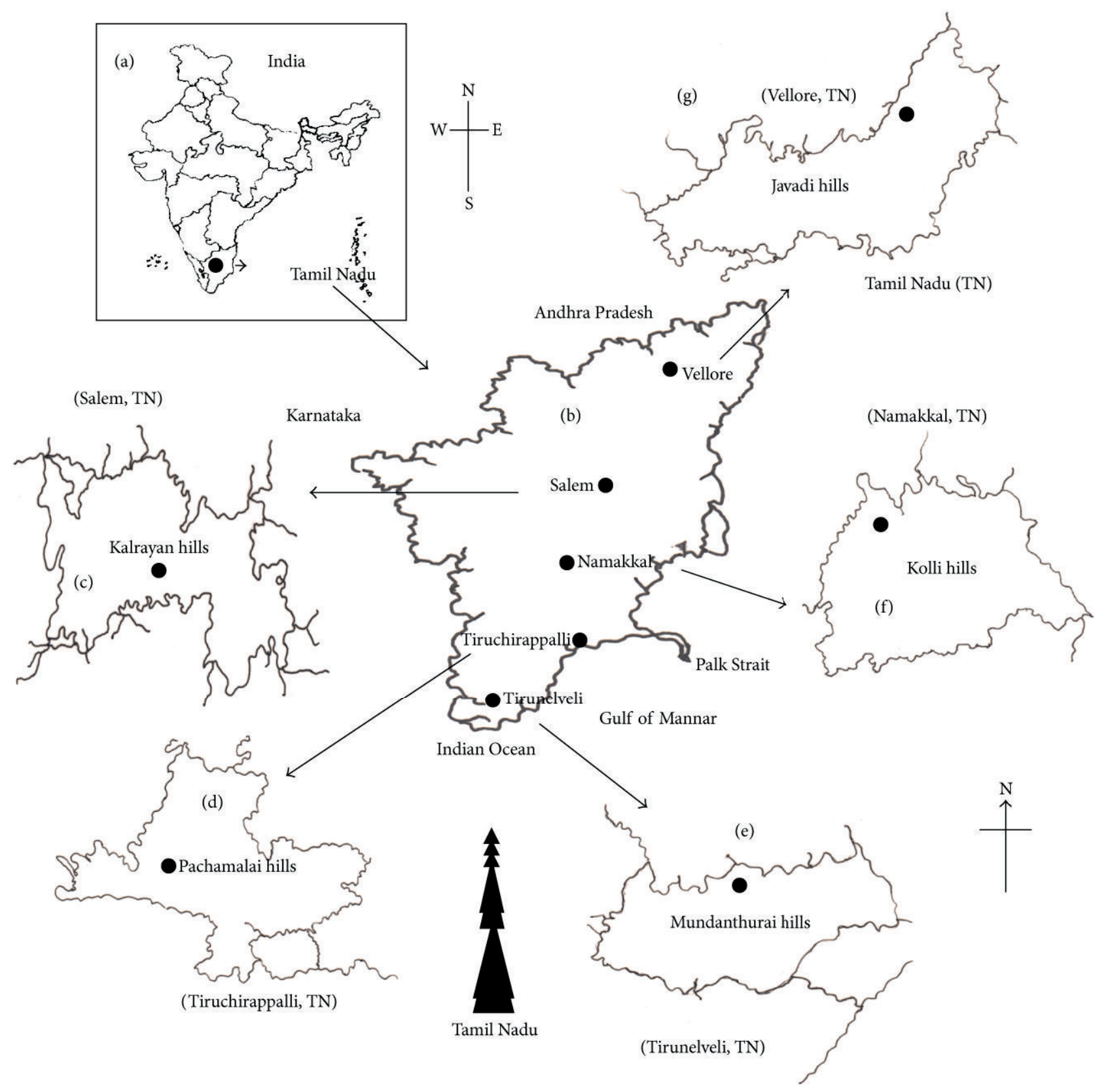

Figure 1: (a) The site for collection of medicinal plants in Western and Eastern Ghats of Tamil Nadu. (b) The landmark (map) of traditional medicine distribution and collection of different types of plants. (c) District map showing the collection site of plants from Kalrayan hills (Salem), (d) Pachamalai hills (Thiruchirappalli), and (e) Mundanthurai (Tirunelveli) rich biodiversity hot-spot of the Western Ghats. (f) Kolli hills (Namakkal), (g) Javadi hills (Vellore), part of the Eastern Ghats, which is a mountain range that runs mostly parallel to the east coast of South India.

a large number of wild edible fruits, including yielding plants such as Citrus acida, Ficus benghalensis, Ficus microcarpa, Ficus racemosa, Phyllanthus emblica, Solanum trilobatum, and Syzygium cumini are popularly used by the tribes.

3.4. Plants Used for Snakebite Treatment. Thirty-four plants used for snakebite treatment are documented (Table 1). Snakebite is a major health hazard that leads to high mortality in tribal settlements. The majority of the antidotes are prepared freshly from plant materials frequently collected from the leaves of A. paniculata, A. echioides, Aristolochia indica, E. alba, E. prostrata, M. pudica, O. sanctum, T. involucrata, and Cleistanthus collinus (Oduvanthalai); the whole plants of Achyranthes aspera and Wedelia calendulacea; the stem-barks and nuts of Strychnos nux-vomica; the roots of Hemidesmus indicus, Tephrosia purpurea, Rauwolfia serpentina, C. roseus, and so forth, and the tubers of Gloriosa superba. The tuber paste is usually applied externally on 
TABLE 1: Some of the important traditional medicinal plant species, families, voucher specimens, parts used, yield of extracts, phytochemical screening, and toxicity on human macrophage cells.

\begin{tabular}{|c|c|c|c|c|c|}
\hline Scientific name & Family & Voucher specimen & Plant parts & Yield (gm) & $\begin{array}{l}\text { Phytochemical } \\
\text { analysis }\end{array}$ \\
\hline Adhatoda vasica Nees & Acanthaceae & D2020 & Leaf & 6.4 & Vasicine \\
\hline $\begin{array}{l}\text { Aegle marmelos (L.) } \\
\text { Correa ex Roxb. }\end{array}$ & Rutaceae & D2018 & Root-bark & 5.8 & Alkaloids \\
\hline $\begin{array}{l}\text { Alangium salvifolium (L.) } \\
\text { f. Wangerin. }\end{array}$ & Alangiaceae & 0140 & Leaf & 6.3 & Phenolic \\
\hline $\begin{array}{l}\text { Andrographis paniculata } \\
\text { Wallich ex Nees }\end{array}$ & Acanthaceae & 0061 & Leaf & 6.8 & Andrographolide \\
\hline $\begin{array}{l}\text { Andrographis echioides } \\
\text { Nees }\end{array}$ & Acanthaceae & 0116 & Leaf & 7.0 & Terpenoids, \\
\hline Acalypha indica L. & Euphorbiaceae & 29644 & Leaf & 6.1 & Acalyphe \\
\hline Acalypha lanceolata L. & Euphorbiaceae & 15791 & Leaf & 7.1 & Alkaloids \\
\hline Achyranthes aspera L. & Amaranthaceae & 2666 & Leaf & 2.7 & Betaine \\
\hline Ageratum conyzoides $\mathrm{L}$. & Asteraceae & 4812 & Leaf & 3.7 & Essential oils \\
\hline Asteracantha longifolia L. & Acanthaceae & 0234 & Stem & 7.4 & Glycosides \\
\hline Azadirachta indica A. Juss. & Meliaceae & D0204 & Leaf, bark & 6.7 & Tannins \\
\hline Borassus flabellifer L. & Arecaceae & D0202 & Root & 0.8 & $\begin{array}{l}\text { Flavonoids, } \\
\text { phenolics }\end{array}$ \\
\hline Boerhavia erecta $\mathrm{L}$. & Nyctaginaceae & 10897 & Whole plant & 2.8 & Phenolics \\
\hline $\begin{array}{l}\text { Calotropis procera (Ait.) } \\
\text { Ait. f. }\end{array}$ & Asclepiadaceae & D073 & Root-bark & 1.3 & Terpenoids \\
\hline $\begin{array}{l}\text { Calotropis gigantea (L.) } \\
\text { R.Br.ex Ait }\end{array}$ & Asclepiadaceae & D070 & Milky latex & 4.8 & Alkaloids \\
\hline Cassia auriculata L. & Caesalpiniaceae & 0141 & Leaf & 4.9 & Saponins \\
\hline Cassia occidentalis L. & Caesalpiniaceae & 0111 & Root & 9.5 & $\begin{array}{l}\text { Flavonoids, } \\
\text { saponins }\end{array}$ \\
\hline Cassia tora $\mathrm{L}$. & Caesalpiniaceae & 0100 & Stem, bark & 6.9 & Saponins \\
\hline Cassia fistula L. & Caesalpiniaceae & 037 & Whole plant & 7.9 & Saponins \\
\hline $\begin{array}{l}\text { Cardiospermum } \\
\text { halicacabum L. }\end{array}$ & Sapindaceae & 0125 & Whole plant & 5.8 & Flavonoids \\
\hline $\begin{array}{l}\text { Catharanthus roseus (L.) } \\
\text { G.Don. }\end{array}$ & Apocynaceae & 0029 & $\begin{array}{l}\text { Leaf, } \\
\text { root }\end{array}$ & $\begin{array}{l}1.4 \\
0.7\end{array}$ & Alkaloids \\
\hline $\begin{array}{l}\text { Cinnamomum zeylanicum } \\
\text { Garcin ex Blume }\end{array}$ & Lauraceae & 00209 & Bark & 3.1 & $\begin{array}{l}\text { Essential oil, } \\
\text { Tannin }\end{array}$ \\
\hline $\begin{array}{l}\text { Cinnamomum iners } \\
\text { Reinw. ex Blume }\end{array}$ & Lauraceae & 043-c & Leaf, bark & & Alkaloids \\
\hline $\begin{array}{l}\text { Cissus quadrangularis } \\
\text { Roxb. }\end{array}$ & Vitaceae & D02023 & Leaf & 6.8 & Glycosides \\
\hline Citrus acida Roxb. Hook.f. & Rutaceae & 0213 & Leaf & 5.2 & $\begin{array}{l}\text { Saponins, } \\
\text { Terpenoids }\end{array}$ \\
\hline Centella asiatica (L.) & Umbelliferae & 0138 & Whole plant & 8.9 & $\begin{array}{l}\text { Flavonoids, } \\
\text { Alkaloids }\end{array}$ \\
\hline $\begin{array}{l}\text { Clerodendrum inerme (L.) } \\
\text { Gaertn. }\end{array}$ & Verbenaceae & D02043 & Stem & 7.8 & Sterols, diterpenes \\
\hline Clitoria ternatea $\mathrm{L}$. & Papilionaceae & D02026 & Seed & 9.8 & Protein \\
\hline $\begin{array}{l}\text { Cleistanthus collinus } \\
\text { (Roxb.) Benth. and } \\
\text { Hook.f. }\end{array}$ & Euphorbiaceae & 0011 & Whole plant & 0.03 & $\begin{array}{l}\text { Cleistanthin, } \\
\text { collinusin }\end{array}$ \\
\hline Cleome gynandropsis L. & Capparidaceae & 12247 & Leaf & 6.2 & Glycosides \\
\hline Cleome viscose $\mathrm{L}$ & Capparidaceae & 29999 & Leaf & 2.7 & Phenolics \\
\hline
\end{tabular}


TABle 1: Continued.

\begin{tabular}{|c|c|c|c|c|c|}
\hline Scientific name & Family & Voucher specimen & Plant parts & Yield (gm) & $\begin{array}{l}\text { Phytochemical } \\
\text { analysis }\end{array}$ \\
\hline Coccinia grandis $\mathrm{W} \& \mathrm{~A}$ & Cucurbitaceae & D02030 & Leaf, root & 0.9 & Glycosides \\
\hline $\begin{array}{l}\text { Cymbopogon citratus } \\
\text { (DC.) }\end{array}$ & Gramineae & D012 & Root & 0.25 & Essential oil \\
\hline Datura metel L. & Solanaceae & D02038 & Leaf, stem & 3.9 & Steroids \\
\hline Eucalyptus globulus Labill. & Myrtaceae & D0220 & Leaf & 1.2 & Terpenoids \\
\hline Eclipta alba (L.) Hassk & Asteraceae & D028 & Whole plant & 0.7 & Phenolic \\
\hline Euphorbia hirta Linn & Euphorbiaceae & $0018-\mathrm{c}$ & Whole plant & 0.12 & - \\
\hline Eclipta prostrata (L.) & Asteraceae & D210 & Leaf & 1.10 & $\begin{array}{l}\text { Triterpenoid, } \\
\text { saponin }\end{array}$ \\
\hline $\begin{array}{l}\text { Eugenia caryophyllus } \\
\text { (Sprengel) Bullock \& } \\
\text { Harrison }\end{array}$ & Myrtaceae & 0025 & Flower buds & 1.16 & Essential oils \\
\hline $\begin{array}{l}\text { Elettaria cardamomum } \\
\text { White et Mason }\end{array}$ & Zingiberaceae & 0009 & Fruit pods & 3.17 & Essential oils \\
\hline Gloriosa superba L. & Liliaceae & $020-\mathrm{S}$ & Tuber & 1.08 & Alkaloids, phenol \\
\hline Jatropha curcas L. & Euphorbiaceae & 015 & Whole plant & 5.3 & $\begin{array}{l}\text { Alkaloids, } \\
\text { flavonoids }\end{array}$ \\
\hline Hyptis suaveolens (L.) Poit. & Lamiaceae & 24688 & Leaf & 6.3 & Essential oil \\
\hline Hemidesmus indicus L. & Asclepiadaceae & D-009 & Roots & & Coumarins \\
\hline $\begin{array}{l}\text { Ichnocarpus frutescens } \\
\text { (L.) R.Br. }\end{array}$ & Apocynaceae & 0110 & Root, flower & 7.3 & Terpenoids \\
\hline $\begin{array}{l}\text { Leucas aspera (Willd.) } \\
\text { Link }\end{array}$ & Labiatae & 0114 & Leaf & 8.3 & Triterpenes \\
\hline Lawsonia inermis L. & Lythraceae & T261 & Leaf & 0.9 & $\begin{array}{l}\text { Glycosides, } \\
\text { phenolic }\end{array}$ \\
\hline $\begin{array}{l}\text { Madhuca longifolia (L.) JF } \\
\text { Macbr }\end{array}$ & Sapotaceae & D01415 & Nut & 9.3 & Sitosterol \\
\hline $\begin{array}{l}\text { Merremia hastate L. } \\
\text { (Desr.) Hallier.f. }\end{array}$ & Convolvulaceae & 10894 & Whole plant & 4.0 & Alkaloids \\
\hline Mentha piperita L. & Lamiaceae & $0217-\mathrm{c}$ & Whole plant & 0.7 & Essential oils \\
\hline Morinda tinctoria Roxb & Rubiaceae & 0122 & Leaf & 1.4 & Glycosides \\
\hline Mimosa pudica L. & Mimosaceae & 0071 & Whole plant & 0.6 & - \\
\hline Oldenlandia umbellata L. & Rubiaceae & D02047 & Leaf & 4.4 & Alkaloids \\
\hline Ocimum sanctum L. & Lamiaceae & 0016 & Whole plant & 3.0 & Alkaloids \\
\hline $\begin{array}{l}\text { Piper attenuatum Buch. } \\
\text { Hamex Miq. }\end{array}$ & Piperaceae & 007 & Flower & 4.6 & Alkaloids \\
\hline $\begin{array}{l}\text { Plumbago zeylanica (L.) } \\
\text { Cav }\end{array}$ & Plumbaginaceae & 0121 & Root & 4.8 & Plumbagin \\
\hline $\begin{array}{l}\text { Plectranthus amboinicus } \\
\text { (L.) Spreng. }\end{array}$ & Lamiaceae & 0410 & Whole plant & 1.2 & $\begin{array}{l}\text { Essential oils, } \\
\text { terpenoids }\end{array}$ \\
\hline $\begin{array}{l}\text { Phyllanthus debilis L. } \\
\text { (Klein ex Willd) }\end{array}$ & Euphorbiaceae & 0120 & Whole plant & 4.9 & Polyphenol \\
\hline $\begin{array}{l}\text { Phyllanthus } \\
\text { madraspatensis L. }\end{array}$ & Euphorbiaceae & 0117 & Whole plant & 5.0 & Polyphenol \\
\hline Premna tomentosa Willd. & Verbenaceae & 0129 & Leaf & 5.3 & Diterpenes \\
\hline Rosmarinus officinalis L. & Lamiaceae & 0017 & Root & 0.23 & Essential oils \\
\hline Rauwolfia serpentine L. & Apocynaceae & $020-S$ & Root & 1.15 & Alkaloid \\
\hline $\begin{array}{l}\text { Sebastiania chamaelea (L.) } \\
\text { Muell Arg. }\end{array}$ & Euphorbiaceae & 0034 & Leaf & 1.1 & Polyphenol \\
\hline Solanum trilobatum L. & Solanaceae & D02054 & Leaf, flower & 4.0 & Tannins \\
\hline
\end{tabular}


TABLE 1: Continued.

\begin{tabular}{|c|c|c|c|c|c|}
\hline Scientific name & Family & Voucher specimen & Plant parts & Yield (gm) & $\begin{array}{l}\text { Phytochemical } \\
\text { analysis }\end{array}$ \\
\hline Sphaeranthus indicus L. & Asteraceae & D02060 & Whole plant & 1.0 & Essential oil \\
\hline Swertia chirata (L.) Ham. & Gentianaceae & D0540 & Whole plant & 1.6 & Glycodises \\
\hline Strychnos nux-vomica L. & Loganiaceae & S-22 & Nuts & 0.36 & Alkaloids \\
\hline Tragia involucrata L. & Euphorbiaceae & D068 & Leaves & 1.6 & Shellsol \\
\hline $\begin{array}{l}\text { Tinospora cordifolia } \\
\text { (Willd.) Miers ex Hoof.f \& } \\
\text { Thoms }\end{array}$ & Menispermaceae & 0118 & $\begin{array}{l}\text { Leaf, } \\
\text { root, stem }\end{array}$ & 5.0 & Glycosides, tannins \\
\hline Tridax procumbens L. & Compositae & 10649 & Leaf & 1.8 & Flavonoids \\
\hline $\begin{array}{l}\text { Terminalia arjuna (DC) } \\
\text { W \& A }\end{array}$ & Combretaceae & 033-c & Bark & 8.0 & Phenolics \\
\hline $\begin{array}{l}\text { Tephrosia purpurea (L.) } \\
\text { Pers }\end{array}$ & Fabaceae & S-43 & Whole plant & 0.8 & Isoflavone \\
\hline Vitex negundo L. & Verbenaceae & 0031 & Leaf & 2.4 & Terpineol \\
\hline Vetiveria zizanioides L. & Gramineae & 0051 & Root & 1.03 & Essential oil \\
\hline $\begin{array}{l}\text { Withania somnifera (L.) } \\
\text { Dunal }\end{array}$ & Solanaceae & D02063 & Root & 2.1 & Alkaloids \\
\hline Wedelia calendulacea Less & Asteraceae & S-24 & Leaves & & Flavonoids \\
\hline Zingiber officinale Rosc. & Zingiberaceae & 0327 & Rhizome & 2.3 & Tannins \\
\hline $\begin{array}{l}\text { Zanthoxylum limonella } \\
\text { (Dennst.) Alston }\end{array}$ & Rutaceae & 009 & Bark & 1.9 & $\begin{array}{l}\text { Alkaloids, essential } \\
\text { oil }\end{array}$ \\
\hline
\end{tabular}

Class of chemical compounds: A: alkaloids, S: saponins, T: tannins, St: steroids, G: glycosides, T: terpenoids, P: polyphenol, P: phenolics, Sh: shellsol, H: hydrocarbon esters.

the site of snakebite, and decoction is given orally for treatment by indigenous people. Besides, these tribes rely on the medicinal plants as ingredients for fabricating a kind of medicated stone for health management. "Vishakallu" (poison stone) is used by the indigenous groups called Kani in Kerala, India, to treat a snakebite. When the stone is placed directly on the bitten area, it sticks to the body to absorb the poison and then become detached when absorption seems to be complete. The ingredients of Vishakallu stones are made with leaves of Ocimum sanctum, Anisomeles malabarica, Leucas aspera, Piper betle, Santalum album, and the pebbles collected from the river bank.

3.5. Survey of Medicinal Plants and Their Health Care Values. The present study is an attempt to provide scientific basis and obtain justification for the traditional beliefs of reliance on a rich diversity of ethnomedicinal plants, along with the rich heritage of traditional medicine practices related to health care system made available by the primitive tribal communities located at different settlements. The native traditional practitioners called "vaidyars" have a good knowledge about the traditional plants locally available for treatment of various diseases (Figures 3(a)-3(p)). Such traditional medical knowledge is used for preparing home remedies, ill health prevention, and routine health maintenance. This knowledge is also applicable to cover other sectors of social life. During the ethnobotanical survey, the wealth of 78 medicinal plant species used by the indigenous tribal community for various types of health treatment was documented. The botanical names, family names, parts used, chemical constituents, and their application are provided (Table 1).

3.6. Antimicrobial Activity of Crude Extracts. In this study, we reported the antimicrobial screening of methanolic crude extracts of 78 medicinal plants (Table 2). Results revealed that 68 plant extracts displayed potent activities against one or more Gram-positive and -negative bacteria. Of which, Tragia involucrata, Citrus acida, Aegle marmelos, Adhatoda vasica, Calotropis procera, Andrographis paniculata and Mentha piperita, Azadirachta indica, Sphaeranthus indicus, and Elettaria cardamomum showed the highest antibacterial activity against the multidrug resistant $B$. pseudomallei (KHW and TES) and $S$. aureus at $100 \mu \mathrm{g} / \mathrm{mL}$ concentration. The extracts showed pronounced antibacterial activity with their inhibitory zones ranging from 20 to $31 \mathrm{~mm}$ in diameter as compared to the standard drugs chloramphenicol and ceftazidime $(29-33 \mathrm{~mm})$. The majority of the plants demonstrated a powerful antimicrobial potency against the multidrug resistant strains of $B$. pseudomallei (KHW and TES), K. pneumonia, and S. aureus. Approximately, twentyone plant extracts exerted only a weak or moderate effect against the tested bacteria, while the crude extract of 13 plants failed to show any effect at all. Except for the plant extracts of $T$. involucrata, A. lanceolata, A. vasica, and $S$. indicus extracts, the majority of the plant extracts were ineffective against the $V$. damsela infection, fascinatingly, only 11 plants exhibited activity against $P$. aeruginosa, of which $S$. indicus, M. piperita, and C. procera were found to have very strong inhibition of bacteria at the tested concentrations. 
TABLE 2: Antimicrobial activity of methanol extract of aromatic medicinal plants and essential oils evaluated against multidrug resistant (MDR) human pathogens at $100 \mu \mathrm{g} / \mathrm{mL}$ concentration.

\begin{tabular}{|c|c|c|c|c|c|c|c|c|c|c|}
\hline \multirow{2}{*}{ Scientific name } & \multicolumn{10}{|c|}{ Microorganisms; growth inhibition zones (6 millimeter in diameters) } \\
\hline & KHW & TES & K.p & K.pr & P. $a$ & S. $a$ & St.p & S.p & V.d & V.d \\
\hline Adhatoda vasica Nees & 28 & 19 & 22 & 18 & 12 & - & 10 & 17 & 16 & - \\
\hline Aegle marmelos (L.) Correa ex Roxb. & 29 & 17 & 9 & 10 & - & 15 & - & - & - & - \\
\hline Alangium salvifolium (L.) f. Wangerin. & 15 & 10 & 8 & 8 & - & 12 & 9 & 10 & - & - \\
\hline Andrographis echioides L. & 12 & 9 & - & - & - & 17 & 8 & 9 & - & - \\
\hline Andrographis paniculata Wallich ex Nees & 26 & 21 & 19 & 13 & - & 25 & 8 & 16 & 12 & - \\
\hline Acalypha indica L. & - & - & - & - & - & 18 & - & - & - & - \\
\hline Acalypha lanceolata $\mathrm{L}$. & - & - & 12 & 13 & - & 10 & - & - & 21 & - \\
\hline Achyranthes aspera L. & - & - & 7 & 14 & - & 15 & 12 & 11 & - & - \\
\hline Ageratum conyzoides L. & 10 & 8 & - & - & - & - & - & - & - & - \\
\hline Asteracantha longifolia $\mathrm{L}$. & 16 & 12 & - & - & - & 15 & - & - & - & - \\
\hline Azadirachta indica A. Juss. & 15 & 17 & 21 & 16 & 14 & 23 & 12 & 14 & - & - \\
\hline Borassus flabellifer L. & 9 & 10 & 17 & 8 & - & - & 9 & 10 & - & - \\
\hline Boerhavia erecta L. & - & - & 8 & 9 & 7 & 16 & - & - & - & - \\
\hline Calotropis procera (L.) & - & - & 15 & - & 18 & 28 & 9 & - & - & - \\
\hline Calotropis gigantea (L.) R.Br.ex Ait & 11 & 9 & - & - & - & 20 & - & - & 9 & 8 \\
\hline Cardiospermum halicacabum L. & 23 & - & - & 14 & - & 9 & 19 & - & - & - \\
\hline Catharanthus roseus (L.) G.Don. & 13 & 7 & 12 & 9 & - & 15 & 11 & 8 & - & - \\
\hline Cassia auriculata L. & 17 & 13 & 12 & - & - & 19 & 13 & - & - & - \\
\hline Cassia occidentalis L. & 18 & - & - & - & - & - & - & - & - & - \\
\hline Cassia tora $\mathrm{L}$. & - & - & - & - & - & - & - & - & - & - \\
\hline Cassia fistula $\mathrm{L}$. & - & - & - & - & - & - & - & - & - & - \\
\hline Citrus acida Roxb. Hook.f. & 26 & 22 & - & 12 & & 29 & 9 & 13 & 8 & - \\
\hline Cissus quadrangularis $\mathrm{L}$. & - & - & - & - & - & - & - & - & - & - \\
\hline $\begin{array}{l}\text { Cinnamomum zeylanicum Garcin ex } \\
\text { Blume }\end{array}$ & 14 & 16 & 7 & 20 & - & 22 & 19 & 7 & - & - \\
\hline Cinnamomum iners Reinw. ex Blume & 20 & 16 & - & 15 & - & 16 & 12 & - & - & - \\
\hline Rosmarinus officinalis L. & - & 9 & - & 10 & - & 7 & - & 8 & - & - \\
\hline Centella asiatica (L.) & 9 & 8 & - & - & - & 11 & - & - & - & - \\
\hline Clerodendrum inerme (L.) Gaertn. & 13 & 7 & 12 & - & - & 15 & 11 & 8 & - & - \\
\hline Clitoria ternatea $\mathrm{L}$. & - & - & - & - & - & - & - & - & - & - \\
\hline Clitoria ternatea $\mathrm{L}$. & 16 & 12 & - & 13 & - & 8 & 10 & - & - & - \\
\hline Cleome gynandropsis $\mathrm{L}$. & - & - & 12 & 19 & 11 & - & 9 & 15 & 12 & - \\
\hline Cleome viscose $\mathrm{L}$. & - & - & 8 & 10 & 14 & 20 & - & - & - & - \\
\hline Coccinia grandis $\mathrm{W} \& \mathrm{~A}$ & 9 & 10 & 17 & 8 & - & - & 9 & 10 & - & - \\
\hline Cymbopogon citratus (DC.) & 16 & 18 & - & 17 & - & 14 & - & - & - & - \\
\hline Datura metel L. & - & - & - & - & - & - & - & - & - & - \\
\hline Eclipta alba (L.) Hassk & 20 & - & - & 9 & - & - & 10 & - & - & - \\
\hline Euphorbia hirta Linn & 11 & - & - & - & - & 16 & - & - & - & - \\
\hline Eucalyptus globulus Labill. & - & - & - & 7 & - & - & - & 7 & - & - \\
\hline Eugenia caryophyllus Bullock \& Harrison & - & 7 & - & 11 & - & 9 & - & 8 & - & - \\
\hline Elettaria cardamomum White et Mason & 21 & 20 & 7 & 14 & - & 22 & 12 & 17 & - & - \\
\hline Hyptis suaveolens (L.) Poit. & 8 & 10 & 8 & 7 & - & - & - & - & - & - \\
\hline Ichnocarpus frutescens (L.) W.J. Aiton & - & - & - & - & - & - & - & - & - & - \\
\hline Jatropha curcas L. & - & - & - & 8 & - & 11 & - & 12 & - & 7 \\
\hline Leucas aspera (Willd.) Link & 9 & 10 & 17 & 8 & - & 12 & 9 & 10 & - & - \\
\hline Lawsonia inermis L. & - & - & - & - & - & - & - & - & - & - \\
\hline Madhuca longifolia (L.) JF Macbr & 18 & 16 & 14 & 19 & 12 & - & 8 & 7 & 9 & - \\
\hline
\end{tabular}


TABLe 2: Continued.

\begin{tabular}{|c|c|c|c|c|c|c|c|c|c|c|}
\hline \multirow{2}{*}{ Scientific name } & \multicolumn{10}{|c|}{ Microorganisms; growth inhibition zones (6 millimeter in diameters) } \\
\hline & $K H W$ & TES & K.p & K.pr & P.a & S. $a$ & St.p & S.p & V.d & V.d \\
\hline Merremia hastate L. (Desr.) Hallier.f. & - & - & - & - & - & - & - & - & - & - \\
\hline Morinda tinctoria Roxb & - & - & - & - & - & - & - & - & - & - \\
\hline Mentha piperita L. & 23 & 17 & 26 & 12 & 20 & 25 & 19 & - & - & - \\
\hline Ocimum sanctum L. & 12 & 9 & 11 & 7 & - & 15 & 7 & 8 & - & - \\
\hline Oldenlandia umbellata $\mathrm{L}$. & - & - & - & - & - & 17 & - & - & - & - \\
\hline Piper attenuatum Buch. Hamex Miq. & 13 & 7 & 12 & 21 & - & 17 & 11 & 8 & - & - \\
\hline Plumbago zeylanica (L.) Cav & 9 & 10 & 17 & 8 & - & 12 & 9 & 10 & - & - \\
\hline Plectranthus amboinicus (L.) Spreng. & - & - & - & 8 & 11 & 15 & - & - & - & - \\
\hline Phyllanthus debilis L. (Klein ex Willd) & 7 & 8 & - & 9 & - & 9 & 18 & 7 & - & - \\
\hline Phyllanthus maderaspatensis $\mathrm{L}$. & 17 & - & - & - & - & - & - & - & - & - \\
\hline Premna tomentosa Willd. & 13 & 10 & - & 15 & - & 9 & 10 & - & - & - \\
\hline Gloriosa superba L. & 17 & 16 & 7 & 8 & - & 15 & - & - & - & 8 \\
\hline Sebastiania chamaelea (L.) Muell Arg. & 19 & 12 & - & 17 & - & 13 & 19 & 8 & - & - \\
\hline Solanum trilobatum $\mathrm{L}$. & - & - & 13 & 9 & - & 8 & - & - & - & - \\
\hline Sphaeranthus indicus L. & 20 & 18 & 21 & 7 & 22 & - & 11 & 9 & - & 16 \\
\hline Swertia chirata (L.) Ham. & - & - & - & - & - & - & - & - & - & - \\
\hline Terminalia arjuna (W. \& A) & - & - & - & - & - & 16 & - & - & - & - \\
\hline $\begin{array}{l}\text { Tinospora cordifolia (Willd.) Miers ex } \\
\text { Hoof.f \& Thoms }\end{array}$ & - & - & 23 & 16 & - & 12 & 15 & - & - & - \\
\hline Tridax procumbens $\mathrm{L}$. & 9 & 8 & 7 & - & - & 14 & - & - & - & - \\
\hline Tragia involucrata $\mathrm{L}$. & 25 & 23 & 20 & - & - & 31 & 28 & 22 & 19 & - \\
\hline Vitex negundo L. & - & - & - & - & - & 14 & - & - & - & - \\
\hline Vetiveria zizanioides (L.) & 11 & 9 & 7 & 7 & - & 16 & - & - & & 8 \\
\hline Withania somnifera (L.) Dunal & 12 & 20 & - & - & - & 15 & 12 & - & - & - \\
\hline Zingiber officinale Rosc. & 14 & 11 & 7 & 15 & - & 7 & 12 & 7 & - & - \\
\hline Zanthoxylum limonella (Dennst.) Alston & 13 & 7 & 12 & 9 & - & - & 11 & 8 & - & - \\
\hline Chloramphenicol (30 $\mu \mathrm{g} / \mathrm{disc})$ & 21 & 12 & 15 & 17 & 29 & 16 & 15 & 18 & 13 & 11 \\
\hline Ceftazidime $(30 \mu \mathrm{g} /$ disc $)$ & 33 & 16 & 22 & 19 & 16 & 25 & 21 & 20 & 12 & 15 \\
\hline
\end{tabular}

${ }^{*}$ Bacteria (+/-). Results obtained in the disc diffusion assay; antibacterial activity is expressed as the mean \pm SD $(n=3)$, of the inhibition by the extract and its diameter around the discs. One way analysis of variance was performed (mean $\pm \mathrm{SD}, n=3$ replicates). Size of inhibition zones were including the sterile blank discs 6 millimeter $(\mathrm{mm})$ in diameters. Absence of bacterial inhibition indicates (-), antibiotic disc $(30 \mu \mathrm{g} / \mathrm{disc})$.

Interestingly, sixteen plants such as Andrographis echioides, C. auriculata, C. viscose, C. gigantea, T. arjuna, Oldenlandia umbellata, Boerhavia erecta, and E. hirta exerted a strong activity against the Gram-positive $S$. aureus bacteria.

3.7. Phytochemical Screening of Plants. The results obtained from the phytochemical screening as shown in Table 1 indicate the presence of various types of secondary metabolites such as polyphenols, tannins, saponins, alkaloids, and glycosides/polysaccharides. Most of the plant extracts relatively rich in alkaloids, phenols, flavonoids, polyphenols, tannins, sterols, and terpenoids were found to inhibit the growth of organisms.

3.8. Antimicrobial Activity of Fractioned Compounds. Active components were purified from the most active extracts for further testing. The compound shellsol of T. involucrata and $C$. acida exhibited the most potent action against the antibiotic resistant strains of $B$. pseudomallei (KHW), S. aureus, B. pseudomallei (TES), and K. pneumoniae. A. marmelos was also found to inhibit the growth of $B$. pseudomallei (KHW) more effectively than other tested bacteria. $A$. vasica showed the broad spectrum growth inhibitory activity on B. pseudomallei (KHW), K. pneumoniae, K. pneumoniae, resistant B. pseudomallei (TES), S. pyogenes, and $V$. damsela. However, E. cardamomum displayed antimicrobial activity on some of the B. pseudomallei (KHW), S. pyogenes, $B$. pseudomallei (TES), and S. typhi strains. Similarly, A. indica exerted the growth inhibition on $K$. pneumoniae and $S$. aureus. Remarkably, Sebastiania chamaelea was more active against K. pneumoniae, K. pneumoniae, and S. pyogenes. The compound from $S$. indicus inhibited the growth of $K$. pneumoniae, K. pneumoniae, B. pseudomallei (KHW and TES), and S. typhi strains, as compared to the activity shown 


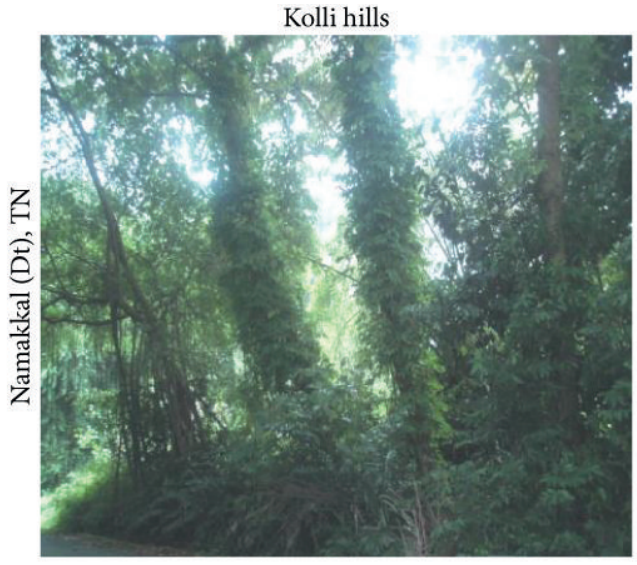

(a)

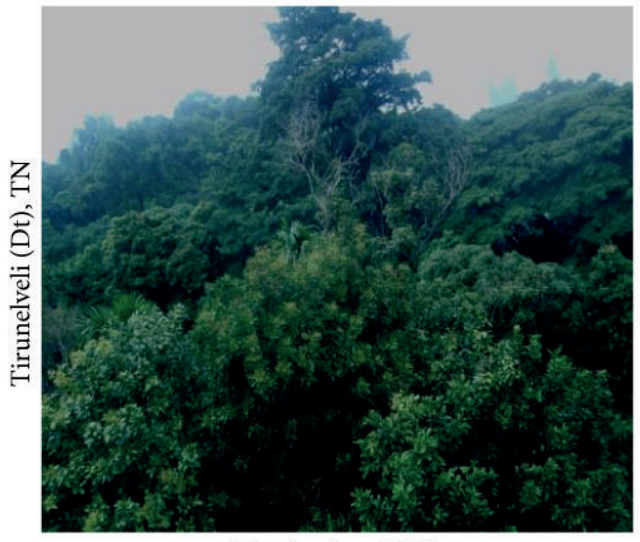

Mundanthurai hills

(b)

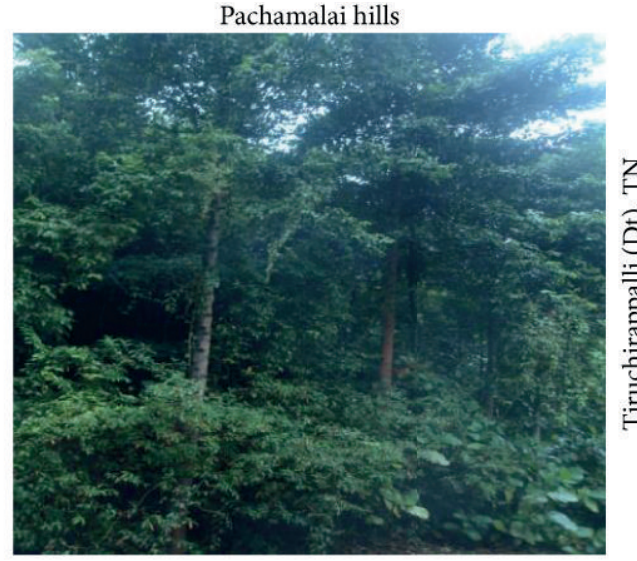

(c)

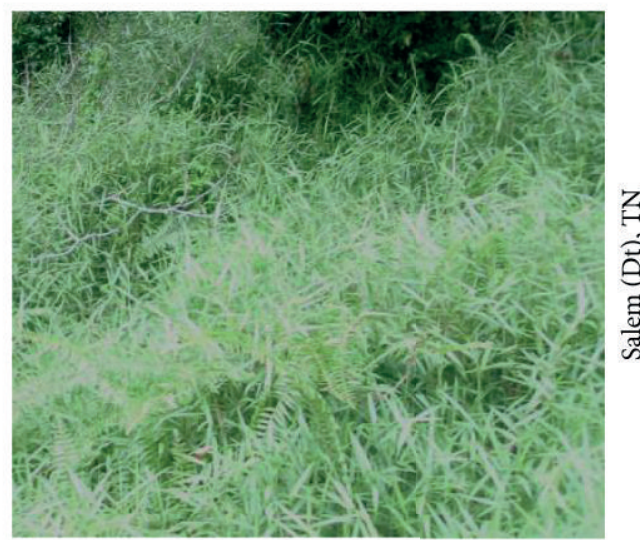

Kalrayan hills

(d)

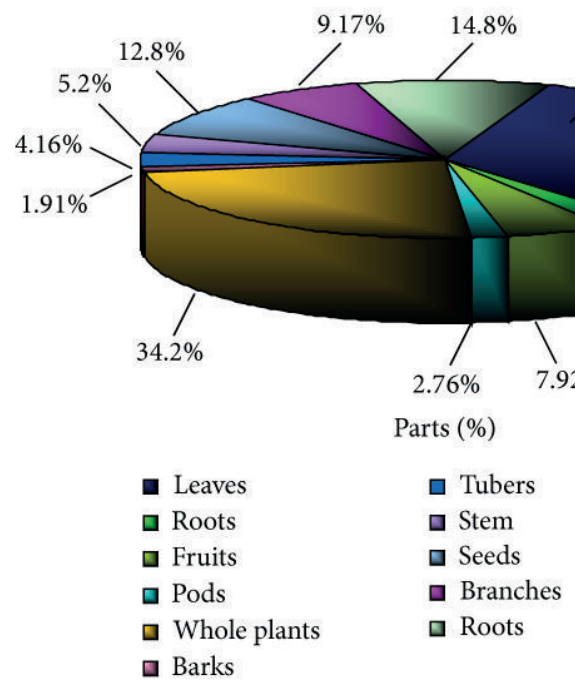

(e)

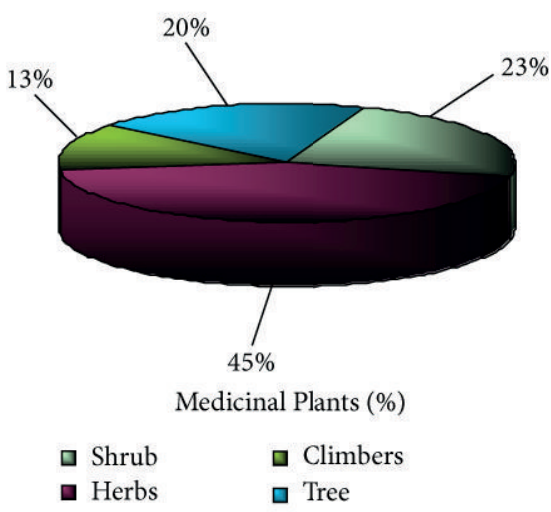

(f)

FIGURE 2: Diverse biodiversity richness of medicinal plants in Western and Eastern Ghats. (a) Topography of plant covering area in Kolli hills (Namakkal district, Tamil Nadu). (b) Aerial view of occurrence of medicinal plants in Mundanthurai hills (Tirunelveli district, TN). (c) Pachamalai hills (Trichy district) and its natural vegetation inhabitants for Malaiyali tribes. (d) Deforestation of natural herbal resources due to urbanization in Kalrayan hills (Salem district) in the Eastern Ghats of TN. (e) Medicinal plants and its various parts used by the natives (traditional healers) for the treatment of diverse human illness with a very high percentage of leaves and whole plants often used for herbal drug preparation by the local practitioners. (f) Various category of plants like shrub, herb, climbers and tree, and the parts used in medicine. 


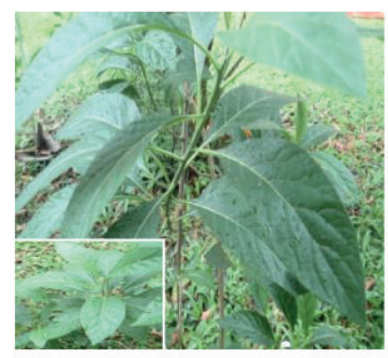

(a)

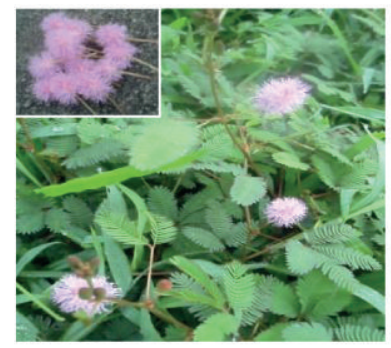

(c)

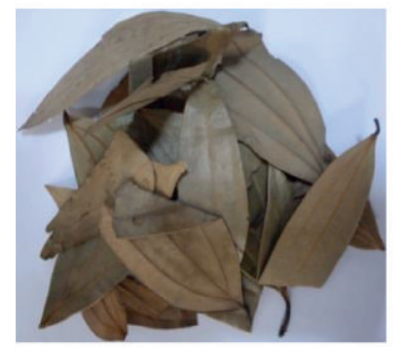

(i)

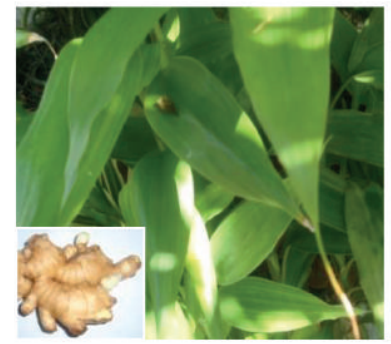

(k)

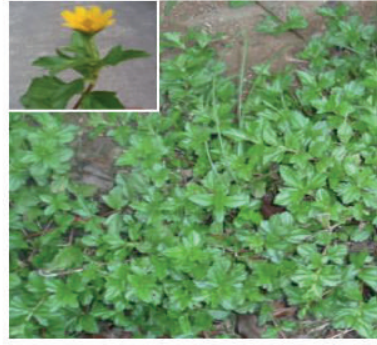

(b)

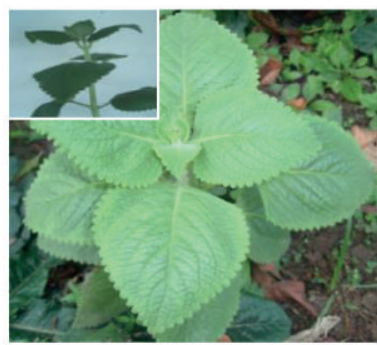

(d)

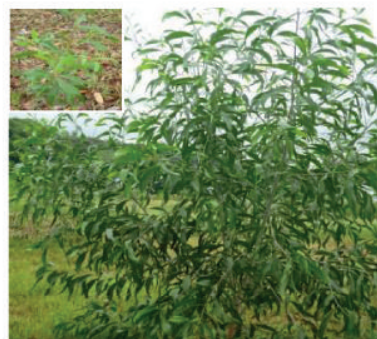

(j)

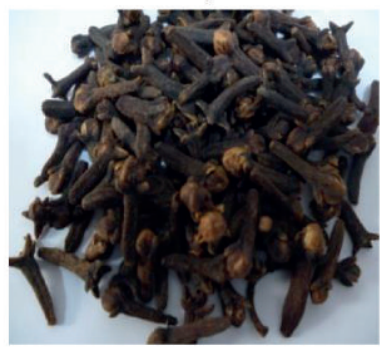

(1)

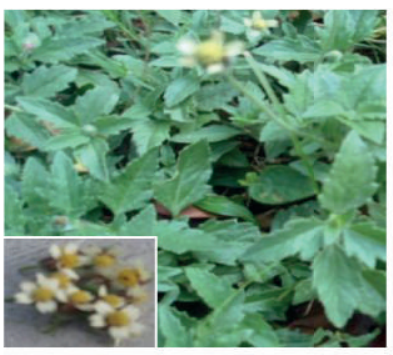

(e)

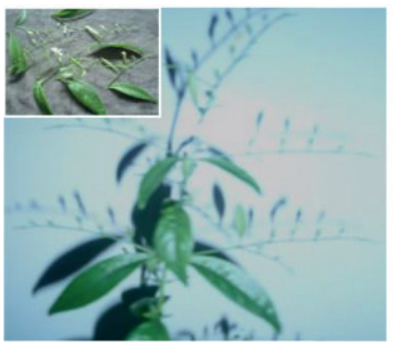

(g)

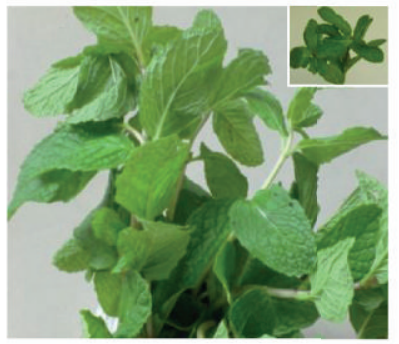

(m)

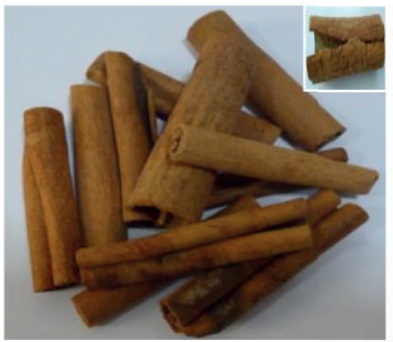

(o)

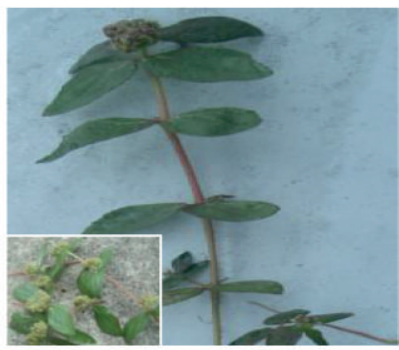

(f)

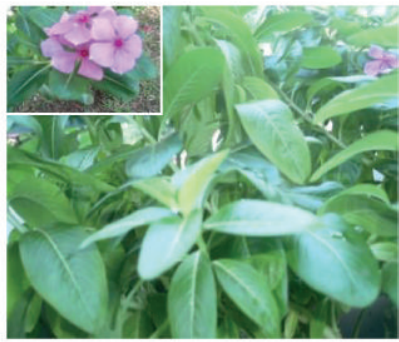

(h)

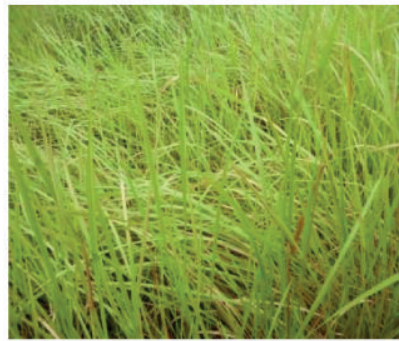

(n)

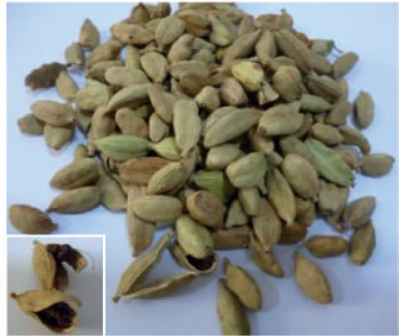

(p)

FIGURE 3: Medicinal aromatic herbs, spices, and toxic plants were collected from the tribal areas of the Western and Eastern Ghats region in Tamil Nadu, India. (a) A. vasica Nees (leaf), (b) Eclipta alba (L.) Hassk. (whole plant), (c) Mimosa pudica L. (whole plant), (d) P. amboinicus (L.) Spreng. (whole plant), (e) T. procumbens (L.) (whole plant), (f) Euphorbia hirta Linn (whole plant), (g) A. paniculata Wallich ex Nees, (H) C. roseus (L.) G.Don. (whole plant) used for therapy. (i) Cinnamomum iners Reinw. ex Blume (leaf) (j) E. globulus Labill. (leaf and bark), (k) Z. officinale Rosc. (Rhizome), (l) E. caryophyllus (Sprengel) Bullock and Harrison (flower buds), (m) M. piperita L. (whole plant), (n) C. citratus (DC.) Clitoria ternatea L. (whole plant), (o) C. zeylanicum Garcin ex Blume (bark), (p) Elettaria cardamomum White et Mason (fruit pod) used for medicine and food preparation.

by the crude extracts (Figure 4). The antimicrobial efficacy of fractions collected from the oil yielding plants was also compared with that of the tested compounds. C. zeylanicum and $R$. officinalis were the most sensitive in controlling the growth of B. pseudomallei (KHW), S. aureus, K. pneumonia, and S. pneumoniae. Fascinatingly, all the compounds obtained from aromatic plants, except those from E. globules, were found to be very effective against the multidrug resistant human pathogen B. pseudomallei (KHW) that causes melioidosis.
On the other hand, compounds from $C$ citrates, O. sanctum, E. caryophyllus, and Z. zizanioide, showed some promising effect only against $S$. aureus (Figure 5). The activity of the compounds were pronounced more than that of the oil yielding plant fractions.

3.9. Minimum Inhibitory Concentrations (MICs). The antibiotic potential of the purified fractions was obtained from the MIC determination. The hydrocarbon ester shellsol 


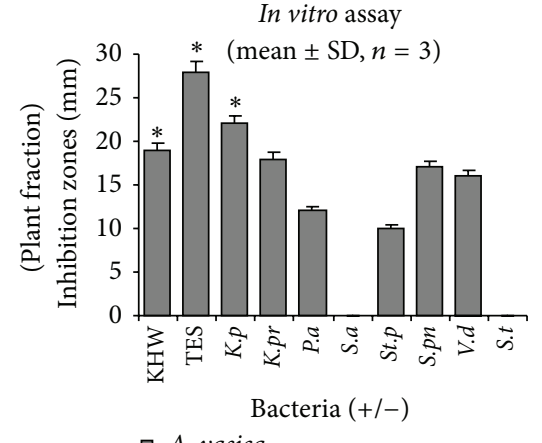

- A. vasica

(a)

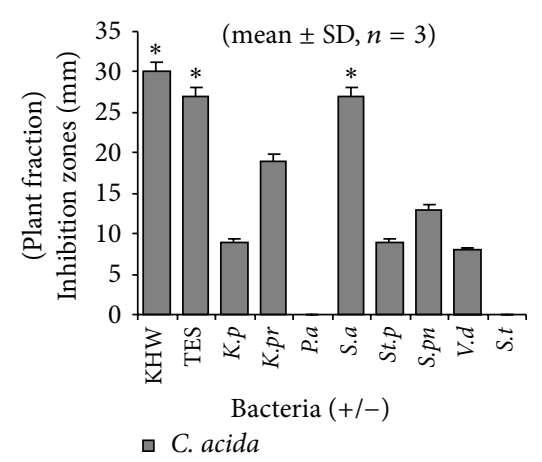

(d)

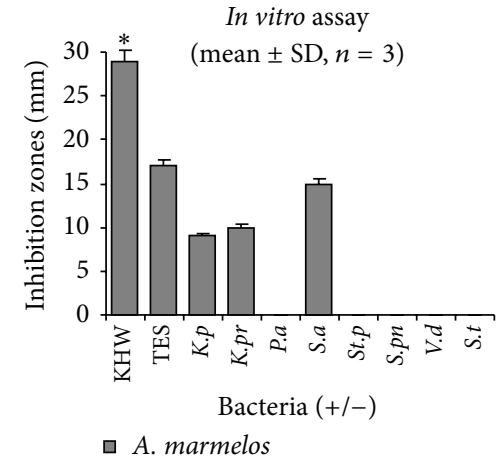

(b)

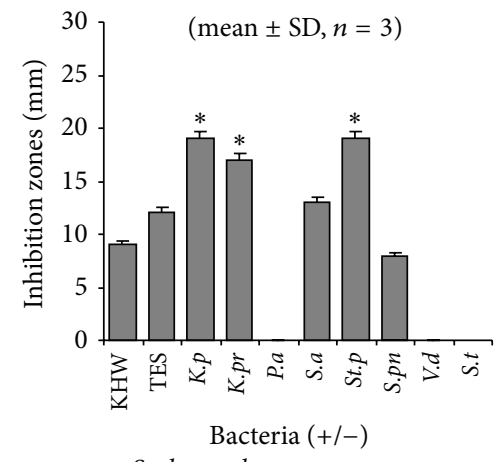

- S. chamaelea

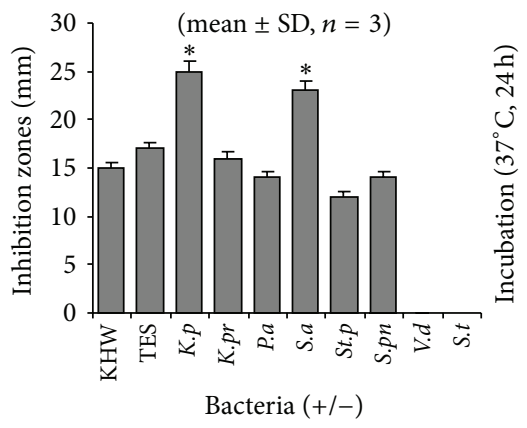

- A. indica

(c)

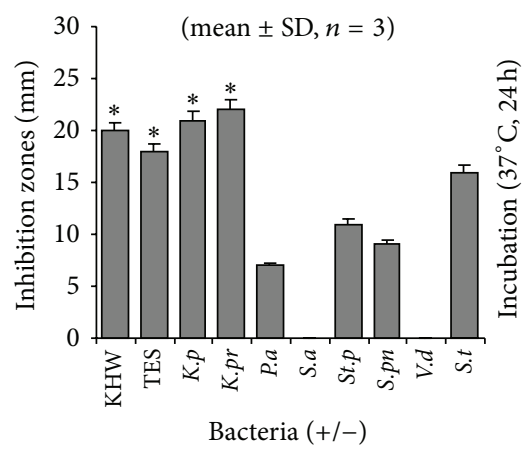

- S. indicus

(f)

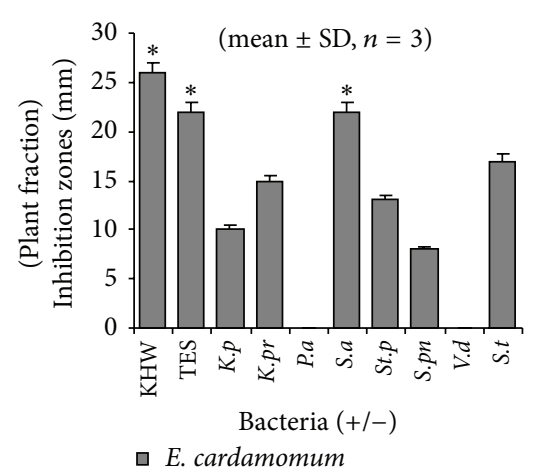

(g)

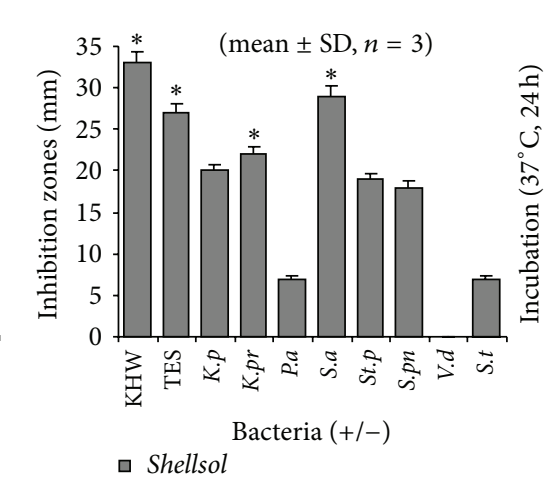

(h)

FIGURE 4: In vitro antimicrobial activity of purified fractions from the most active plant extracts tested against bacteria. Growth inhibition zones were measured and analyzed with mean \pm standard deviation $(\mathrm{SD}),(n=3)$ using one way analysis of variance. Level of significance at $\left({ }^{*} P>0.01\right)$. Most of the fractions exerted a potent inhibitory effect against multidrug resistant Gram-negative bacteria (B. pseudomallei strains KHW and TES), K. pneumonia, and Gram-positive bacteria S. aureus.

(T. involucrata) and C. acida showed an interesting inhibitory potential against S. aureus (MIC of $7.8 \mu \mathrm{g} / \mathrm{mL}$ ) and B. pseudomallei strain of KHW (MIC of $15.6 \mu \mathrm{g} / \mathrm{mL}$ ). A. vasica showed an MIC of $15.6 \mu \mathrm{g} / \mathrm{mL}$ against B. pseudomallei (KHW) and an MIC of $31.25 \mu \mathrm{g} / \mathrm{mL}$ against K. pneumoniae, $K$. pneumoniae, $S$. pyogenes, and $V$. damsela strains. Fractions from $A$. marmelos and terpenoid from $A$. indica exerted bacteriostatic effect with MIC values of $31.25 \mu \mathrm{g} / \mathrm{mL}$ on some selected bacteria including $B$. pseudomallei of KHW, S. aureus, and B. pseudomallei of TES. The MIC of $31.25 \mu \mathrm{g} / \mathrm{mL}$ was found for E. cardamomum against S. aureus,
K. pneumonia, and S. pyogenes. S. indicus displayed a very strong inhibition against MDR K. pneumoniae (MIC of $15.6 \mu \mathrm{g} / \mathrm{mL}$ ), and against B. pseudomallei (KHW and TES) at MIC of $31.25 \mu \mathrm{g} / \mathrm{mL}$. When the antimicrobial efficacies of purified fractions from aromatic plants were compared, the C. zeylanicum fraction displayed an important antimicrobial effect against S. aureus (MIC of $7.8 \mu \mathrm{g} / \mathrm{mL}$ ), MDR B. pseudomallei of KHW (MIC of $15.6 \mu \mathrm{g} / \mathrm{mL}$ ), and S. pneumoniae (MIC of $31.25 \mu \mathrm{g} / \mathrm{mL}$ ). The essential oil from M. piperatea showed MIC value of $31.25 \mu \mathrm{g} / \mathrm{mL}$ against $K$. pneumoniae, $S$. aureus, and B. pseudomallei (KHW), respectively. O. sanctum 


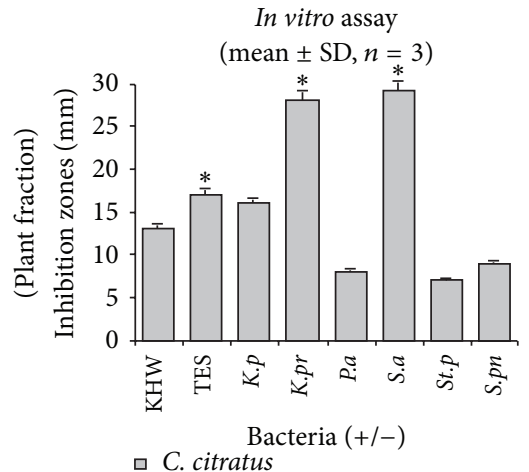

(a)

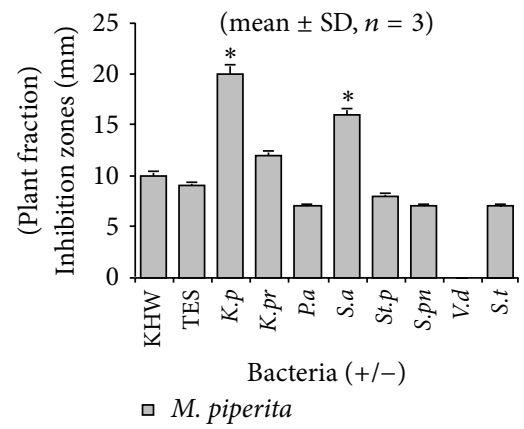

(d)

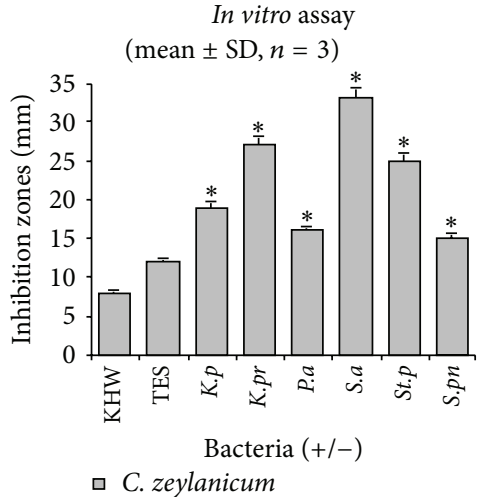

(b)

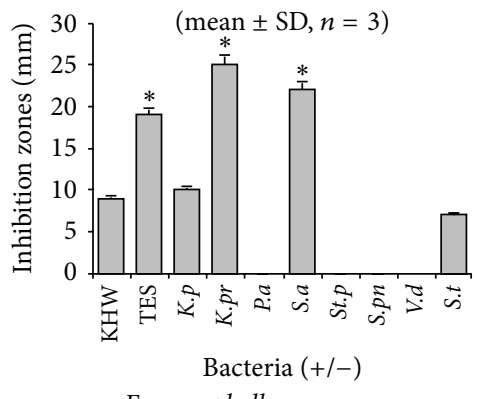

- E. caryophyllus

(e)

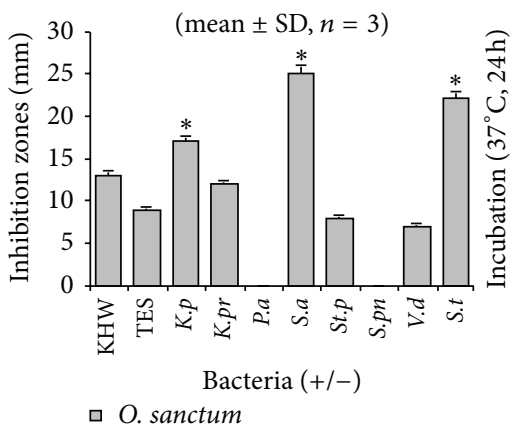

(c)

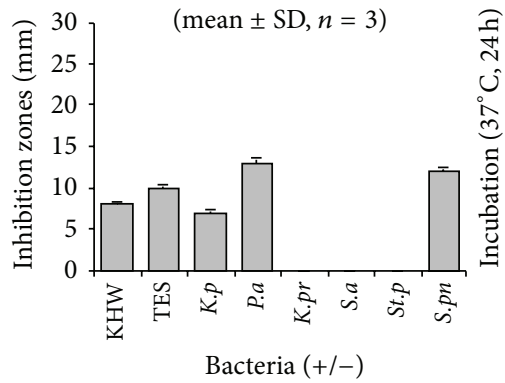

- E. globulus

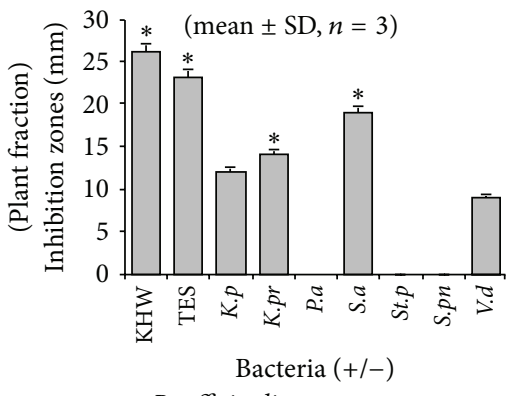

๑ R. officinalis

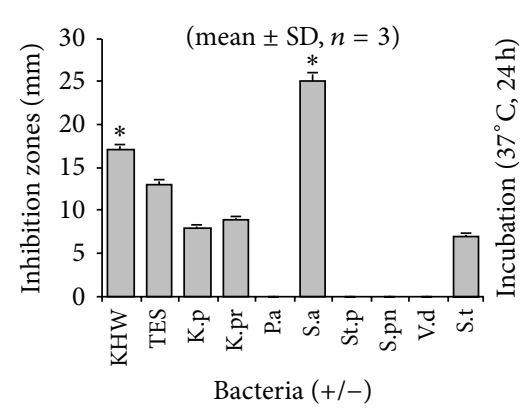

- V. zizanioides

(g)

(h)

Figure 5: Comparison of antimicrobial effect of plant compounds obtained from the most popularly used sources of essential oils as assayed by the disc-diffusion method in vitro. It displayed a powerful activity against B. pseudomallei and S. aureus than the other bacteria strains. Other compounds showed only a moderate or weak action against the tested bacteria. Values for zone of bacterial growth inhibition were presented as mean $\pm \mathrm{SD},(n=3)$ with level of significance at $\left({ }^{*} P>0.01\right)$.

and C. citratus fractions also showed antimicrobial activity (MICs of $31.25-125 \mu \mathrm{g} / \mathrm{mL}$ ) only at higher concentrations against the tested bacteria. In addition to that, higher concentrations $(>250 \mu \mathrm{g} / \mathrm{mL}$ ) (of Vetiveria fractions) were required to inhibit Vibro species, and others (including E. globulus fractions) failed to show any effect at tested concentrations $(7.8-125 \mu \mathrm{g} / \mathrm{mL}$ ). However, the purified fractions (from most active medicinal plants) showed strong bacteriostatic inhibition against the tested organisms (Table 3 ).

3.10. Cytotoxic Effects of Plants. When the components were assayed for cytotoxicity against the normal human skin fibroblasts (HEPK) cells, the compounds obtained from
E. cardamomum, T. involucrata, S. indicus, C. acida, A. vasica, A. marmelos, A. indica, and A. paniculata did not show toxicity up to $1000 \mu \mathrm{g} / \mathrm{mL}$ (see Figures S1 and S2 in Supplementary Material available online at http://dx.doi.org/10.1155/ 2013/525613). A slight reduction of cell proliferation was noted only at higher doses $(2000 \mu \mathrm{g} / \mathrm{mL})$. In contrast, cell proliferation was markedly reduced after exposure of HEPK cells to O. sanctum, E. globulus, V. zizanioides, C. citratus, and E. globulus compounds. There was no gradual reduction in skin cell proliferation seen after exposure to C. zeylanicum, R. officinalis, and M. piperita (see Figures S3 and S4) compounds. The toxicity was found to be concentrationdependent when the skin fibroblasts (HEPK) cells were 
TABLE 3: Minimum inhibitory concentrations (MICs) of purified plant fractions and essential oils against antibiotic resistant bacteria.

\begin{tabular}{|c|c|c|c|c|c|c|c|c|c|c|c|}
\hline \multirow{2}{*}{ Botanical name } & \multirow{2}{*}{ Family } & \multirow{2}{*}{ Parts used } & \multicolumn{9}{|c|}{ Gram-positive and -negative bacteria (MICs $\mu \mathrm{g} / \mathrm{mL}$ ) } \\
\hline & & & $K H W$ & TES & K.p & K.Pr & S. $a$ & St.p & S.p & V.d & S.t \\
\hline A. indica Juss. & Meliaceae & Seed (fraction) & 31.25 & 62.5 & 125 & $<250$ & 31.25 & - & - & - & - \\
\hline A. marmelos (L.) & Rutaceae & Root-bark (F) & 31.25 & 31.25 & - & - & 31.25 & 62.5 & 125 & 250 & - \\
\hline A. paniculata Nees & Acanthaceae & Leaf (fraction) & 250 & - & 125 & - & 62.5 & - & - & 125 & - \\
\hline A. vasica Nees & Acanthaceae & Fraction (Stem) & 15.6 & 31.25 & 31.25 & 62.5 & - & $<250$ & 31.25 & 31.25 & - \\
\hline C. acida Roxb. & Rutaceae & Leaf (fraction) & 15.6 & 62.5 & - & - & 7.8 & - & 31.25 & - & - \\
\hline E. cardamomum White et Mason & Acanthaceae & Fraction (WP) & 62.5 & - & 31.25 & - & 31.25 & 250 & 62.5 & - & - \\
\hline S. indicus (L.) & Euphorbiaceae & Whole plant (F) & 31.25 & 125 & 15.6 & - & 62.5 & - & - & $<250$ & - \\
\hline T. involucrata (L.) & Euphorbiaceae & Shellosol (leaf) & 15.6 & 31.25 & - & - & 7.8 & 62.5 & - & - & - \\
\hline Cinnamomum zeylanicum (L.) & Lauraceae & Bark (fraction) & 15.6 & 62.5 & - & - & 7.8 & 31.25 & - & - & 125 \\
\hline Cymbopogon citratus (L.) & Graminae & Leaf (fraction) & 250 & $<250$ & 62.5 & - & 62.5 & 125 & - & - & - \\
\hline Eugenia caryophyllus (L.) & Myrtaceae & Flower buds (F) & 62.5 & 125 & - & - & 62.5 & - & - & - & - \\
\hline Eucalyptus globulus (L.) & Myrtaceae & Fraction (leaf) & - & - & - & - & - & - & $<250$ & - & $<250$ \\
\hline Mentha piperita (L.) & Labiatae & Fraction (WP) & - & 62.5 & 31.25 & - & 31.25 & - & $<250$ & - & - \\
\hline Ocimum sanctum (L.) & Labiatae & Leaf (fraction) & 62.5 & 125 & - & 31.25 & 62.5 & - & 125 & $<250$ & - \\
\hline Rosmarinus officinalis (L.) & Labiatae & Rosemary oil & 31.25 & - & - & - & 31.25 & - & - & - & - \\
\hline Vetiveria zizanioides (L.) & Graminae & Root (fraction) & & - & 125 & 125 & - & - & - & $>250$ & $>250$ \\
\hline
\end{tabular}

The bacterial growth inhibitory activity was compared for significant differences within the bacterial strains by broth-dilution method at $250,125,62.5,31.25$, 15.6 , and $7.8 \mu \mathrm{g} / \mathrm{mL}$. F: fractions.

exposed to various compounds. The cell proliferation was increased by the influence of the plant components at the lower concentrations. Whereas the oil yielding plant compounds showed inhibition of cell proliferation and toxicity at higher doses $250-1000 \mu \mathrm{g} / \mathrm{mL}$.

\section{Discussion}

The Western Ghats is considered as one of the richest biodiversity hotspots in the world [41]. In this survey, we collected nearly 78 medicinal plants from Western and Eastern Ghats that are edible and popularly used for curing various ailments including snakebite. Traditional remedies have a long-standing history in many tribal settlements in $\mathrm{TN}$, India, and they continue to provide useful and applicable tools for treating ailments [42]. The ingredients that make up the "Vishakallu" stone, which is used as an antidote for snakebite, are different herbs and pebbles available from the river banks. Likewise, aqueous paste and decoction obtained from the leaves of $A$. paniculata are widely used for snakebite treatment by indigenous people [43]. Previous studies have reported that ethnomedicine plays major roles in conserving the disappearing knowledge of tribal communities [44-47]. The traditional beliefs of reliance on a rich diversity of ethnomedicinal plants located at different settlements have also been confirmed in another study $[48,49]$. Herein, we explored the various types of traditional practices reported by the primitive tribal communities with a view to gain further knowledge from such studies.

In the present investigation, potentially rich sources of tribal medicine (71 plants) were scientifically evaluated for their antibacterial activity against the MDR bacteria, and the accumulated data was disseminated for the first time to the scientific community. Out of the 71 medicinal plants screened for the antibacterial activity, 10 of them ( $T$. involucrata, C. acida, A. marmelos, A. vasica, C. procera, A. paniculata and M. piperita, A. indica, S. indicus, and E. cardamomum) displayed the highest antibacterial activity against the multidrug resistant $B$. pseudomallei (KHW and TES) and $S$. aureus strains. The antibacterial activity of those crude plant extracts was as equally effective as that of the standard drugs. Our findings corroborated with the previous reports made on the antistaphylococcal activity of tribal medicinal plants $[34,50,51]$. On the other hand, isolated components from the most active extracts of T. involucrata, shellsol, and C. acida exhibited the most potent action against the antibiotic resistant B. pseudomallei (KHW), K. pneumoniae, and S. aureus strains. These results further confirmed our previous findings on the leaves of T. involucrata and its compounds hydrocarbon ester-like shellsol, which displayed a high antibacterial effect against the different bacterial strains, especially that of S. aureus [34]. Eugenol and caryophyllene are the active agents contained in the M. piperita [52] and O. sanctum plants, which are believed to be mainly responsible for the antimicrobial properties of these plants [53]. Interestingly, the compounds obtained from the aromatic plants such as $C$. zeylanicum and $R$. officinalis were also found to be very effective against the multidrug resistant human pathogen $K$. pneumoniae, S. aureus, S. typhi, and B. pseudomallei (KHW) that causes melioidosis.

The inhibitory potential determined for the shellsol (T. involucrata), vaseline (A. vasica), C. acida, and C. zeylanicum indicates that the MIC of $7.8-31.25 \mu \mathrm{g} / \mathrm{mL}$ found against the $B$. pseudomallei of KHW, K. pneumoniae, $K$. pneumoniae, S. pyogenes, and V. damsel, and S. pneumoniae was quite low. Similarly, lower MIC values were found for 
M. piperita (MIC of $1.13-2.25 \mathrm{mg} / \mathrm{mL}$ ) against the above bacterial strains [52]. MIC was found for the most active alcohol extracts of A. salvifolium (MIC $0.034-0.263 \mathrm{mg} / \mathrm{mL}$ ) on S. aureus [54], and the MIC for S. trilobatum aqueous extracts determined against the tested organisms ranged from 0.06 to $0.5 \mathrm{mg} / \mathrm{mL}$ [55]. C. zeylanicum was found to have an effective antibacterial activity (MIC $64 \mu \mathrm{g} / \mathrm{mL}$ ) against $P$. aeruginosa, E. coli, B. subtilis, and $S$. aureus [56]. Previously, several investigators have demonstrated that active agents exert interesting activity against bacteria even at lower concentrations tested $[57,58]$. The bacteriostatic mechanism involves damage to the cell walls of bacteria, followed by inhibition of protein synthesis that ultimately leads to bacterial death [59]. The most active plants are widely used by various tribes as traditional treatment (i.e., cut wounds, skin infection, and scabies), thus indicating the potential for further development into promising drugs. In order to ascertain the safety and efficacy of the most active compounds, their effect on human skin fibroblast cells was evaluated. Chemical constituents of E. cardamomum, T. involucrata, S. indicus, C. acida, A. vasica, A. marmelos, $A$. indica, and $A$. paniculata plants failed to produce any noticeable toxicity up to $1000 \mu \mathrm{g} / \mathrm{mL}$. Although some of the tested compounds exhibited a slight reduction of cell proliferation and some minor morphological changes, such changes were insignificant at lower doses and became evident only at higher doses. However, certain aromatic compounds of O. sanctum, E. globulus, V. zizanioides, C. citratus, and E. globulus plants showed reduction of cell proliferation against HEPK cells.

Our phytochemical screening also provides evidence of the presence of several types of compounds that are mainly responsible for the remarkable antibacterial effect of these plants. The differences noted for the bactericidal activity of various plant extracts in this study appears to be directly related to the diversity of compounds (shown in parentheses) that are accumulated in the following plants (e.g., A. marmelos and O. umbellate) [60-62]. Compounds like tannins, phenol, and polyphenols can bind the Gramnegative bacteria to form a heavy soluble complex on the cell surface, which subsequently disturbs the availability of receptor on cells and kills the bacteria [63]. Several species having wide spectra of antimicrobial activity mainly due to the active constituents such as essential oil, phenolic compounds like thymol, carvacrol in oregano and thyme, eugenol in clove, and cinnamon were also identified previously $[64,65]$. Essential oils degrade the cell wall, interact with the cell components, and then disrupt the cytoplasmic membrane [66]. The antimicrobial effect of phenolic compounds may involve multiple modes of action, including damage to the membrane protein, interference with membrane integrated enzymes [67], causing leakage of cellular components, coagulation of cytoplasm, depletion of the proton motive force, alteration of fatty acid and phospholipid constituents, impairment of enzymatic mechanisms for production and metabolism, alteration of nutrient uptake and electron transport [68], influencing the synthesis of DNA and RNA, and destroying protein translation and the function of the mitochondrion in eukaryotes [69]. The mode of action of antimicrobial agents depends on the type of microorganism and is mainly related to their cell wall structure and the outer membrane arrangement. Most of the plant spices and herbs contain complex phenolics (i.e., phenolic acids, flavonoids, tannins, lignans, coumarins, quinines). In addition, the mechanisms of action of each phenolic compound against various bacteria are also very complicated [26, 70]. Further investigation is therefore required to understand the relationship between the antimicrobial action and the chemical structure of every phenolic compound in the tested extracts. The information available from previous pharmacological sources combined with the findings herein reported on the medicinal plant extracts may serve as essential data for future drug development to combat diseases caused by the MDR bacterial strains.

\author{
Abbreviations \\ MDR: Multidrug resistant bacteria \\ TES: $\quad$ Strain of Burkholderia pseudomallei \\ MRSA: Methicillin-resistant Staphylococcus aureus \\ VRE: Vancomycin-resistant enterococci \\ MTB: Mycobacterium tuberculosis \\ $\mathrm{CH}_{3} \mathrm{OH}$ : Methanol \\ CFU: Colony forming unit \\ HCl: Hydrochloric acid \\ TLC: Thin layer chromatography \\ $\mathrm{H}_{2} \mathrm{SO}_{4}$ : Sulphuric acids \\ UV: $\quad$ Ultraviolet spectrophotometer \\ HEPK: Human skin fibroblasts.
}

\section{Acknowledgments}

The authors are thankful to the ERI, Loyola College, Chennai, India, for his support during the survey. The authors also thank the Department of Microbiology, NUHS, NUS for the bacterial cultures used in this investigation. The authors are thankful to the tribal people for sharing their healing knowledge on medicinal aromatic plants.

\section{References}

[1] M. N. Alekshun and S. B. Levy, "Molecular mechanisms of antibacterial multidrug resistance," Cell, vol. 128, no. 6, pp. 10371050, 2007.

[2] A. D. Russell, "Antibiotic and biocide resistance in bacteria: introduction," Symposium Series Society for Applied Microbiology, vol. 31, no. 92, pp. 1S-3S, 2002.

[3] D. R. Guay, "Treatment of bacterial skin and skin structure infections," Expert Opinion on Pharmacotherapy, vol. 4, no. 8, pp. 1259-1275, 2003.

[4] A. J. Brosnahan and P. M. Schlievert, "Gram-positive bacterial superantigen outside-in signaling causes toxic shock syndrome," FEBS Journal, vol. 278, no. 23, pp. 4649-4667, 2011.

[5] K. Fellermann, J. Wehkamp, K. R. Herrlinger, and E. F. Stange, "Crohn's disease: a defensin deficiency syndrome?" European Journal of Gastroenterology and Hepatology, vol. 15, no. 6, pp. 627-634, 2003. 
[6] L. Chen, S. Lin, R. Agha-Majzoub, L. Overbergh, C. Mathieu, and L. S. Chan, "CCL27 is a critical factor for the development of atopic dermatitis in the keratin-14 IL-4 transgenic mouse model," International Immunology, vol. 18, no. 8, pp. 1233-1242, 2006.

[7] J. H. Song, V. Thamlikitkul, and P. R. Hsueh, "Clinical and economic burden of community-acquired pneumonia amongst adults in the Asia-Pacific region," International Journal of Antimicrobial Agents, vol. 38, no. 2, pp. 108-117, 2011.

[8] D. A. B. Dance, "Melioidosis," Current Opinion in Infectious Diseases, vol. 15, no. 2, pp. 127-132, 2002.

[9] N. J. White, "Melioidosis," The Lancet, vol. 361, no. 9370, pp. 1715-1722, 2003.

[10] S. Yang, "Melioidosis research in China," Acta Tropica, vol. 77, no. 2, pp. 157-165, 2000.

[11] P. R. Hsueh, L. J. Teng, L. N. Lee et al., "Melioidosis: an emerging infection in Taiwan?" Emerging Infectious Diseases, vol. 7, no. 3, pp. 428-433, 2001.

[12] C. Walsh and S. Fanning, "Antimicrobial resistance in foodborne pathogens-a cause for concern?" Current Drug Targets, vol. 9, no. 9, pp. 808-815, 2008.

[13] M. Vorachit, P. Chongtrakool, S. Arkomsean, and S. Boonsong, "Antimicrobial resistance in Burkholderia pseudomallei," Acta Tropica, vol. 74, no. 2-3, pp. 139-144, 2000.

[14] F. M. Thibault, E. Hernandez, D. R. Vidal, M. Girardet, and J. D. Cavallo, "Antibiotic susceptibility of 65 isolates of Burkholderia pseudomallei and Burkholderia mallei to 35 antimicrobial agents," Journal of Antimicrobial Chemotherapy, vol. 54, no. 6, pp. 1134-1138, 2004.

[15] R. L. S. Sikarwar, "Ethnogynaecological uses of plants new to India," Ethnobotany, vol. 12, no. 1-2, pp. 112-115, 2002.

[16] R. Siva, "Status of natural dyes and dye-yielding plants in India," Current Science, vol. 92, no. 7, pp. 916-925, 2007.

[17] P. Kumar Singh, V. Kumar, R. K. Tiwari, A. Sharma, Ch. V. Rao, and R. H. Singh, "Medico-ethnobotany of 'chatara' block of district sonebhadra, Uttar Pradesh, India," Advances in Biological Research, vol. 4, no. 1, pp. 65-80, 2010.

[18] W. H. Lewis and M. P. Elwin-Lewis, Medical Botany: Plants Affecting Human Health, John Wiley \& Sons, New York, NY, USA, 2003.

[19] R. Perumal Samy, P. N. Pushparaj, and P. Gopalakrishnakone, "A compilation of bioactive compounds from Ayurveda," Bioinformation, vol. 3, no. 3, pp. 100-110, 2008.

[20] World Health Organization (WHO), Traditional Medicine Strategy 2002-2005, Genera: World Health Organization, Geneva, Switzerland, 2002.

[21] P. S. Haddad, G. A. Azar, S. Groom, and M. Boivin, "Natural health products, modulation of immune function and prevention of chronic diseases," Evidence Based Complementary and Alternative Medicine, vol. 2, no. 4, pp. 513-520, 2005.

[22] K. Poonam and G. S. Singh, "Ethnobotanical study of medicinal plants used by the Taungya community in Terai Arc Landscape, India," Journal of Ethnopharmacology, vol. 123, no. 1, pp. 167-176, 2009.

[23] G. B. Mahady, "Medicinal plants for the prevention and treatment of bacterial infections," Current Pharmaceutical Design, vol. 11, no. 19, pp. 2405-2427, 2005.

[24] V. P. Kumar, N. S. Chauhan, H. Padh, and M. Rajani, "Search for antibacterial and antifungal agents from selected Indian medicinal plants," Journal of Ethnopharmacology, vol. 107, no. 2, pp. 182-188, 2006.
[25] R. Nair, T. Kalariya, and S. Chanda, "Antibacterial activity of some plant extracts used in folk medicine," Journal of Herbal Pharmacotherapy, vol. 7, no. 3-4, pp. 191-201, 2007.

[26] D. Kalemba and A. Kunicka, "Antibacterial and antifungal properties of essential oils," Current Medicinal Chemistry, vol. 10, no. 10, pp. 813-829, 2003.

[27] A. H. Ashour, "Antibacterial, antifungal, and anticancer activities of volatile oils and extracts from stems, leaves, and flowers of Eucalyptus sideroxylon and Eucalyptus torquata," Cancer Biology and Therapy, vol. 7, no. 3, pp. 399-403, 2008.

[28] M. J. Abad, L. M. Bedoya, L. Apaza, and P. Bermejo, "The Artemisia L. genus: a review of bioactive essential oils," Molecules, vol. 17, no. 3, pp. 2542-2566, 2012.

[29] H. N. Marzoug, M. Romdhane, A. Lebrihi et al., "Eucalyptus oleosa essential oils: chemical composition and antimicrobial and antioxidant activities of the oils from different plant parts (stems, leaves, flowers and fruits)," Molecules, vol. 16, no. 2, pp. 1695-1709, 2011.

[30] C. Proestos, D. Sereli, and M. Komaitis, "Determination of phenolic compounds in aromatic plants by RP-HPLC and GCMS," Food Chemistry, vol. 95, no. 1, pp. 44-52, 2006.

[31] S. Chao, G. Young, C. Oberg, and K. Nakaoka, "Inhibition of methicillin-resistant Staphylococcus aureus (MRSA) by essential oils," Flavour and Fragrance Journal, vol. 23, no. 6, pp. 444-449, 2008.

[32] M. Akin, D. Oguz, and H. T. Saracoglu, "Antibacterial effects of some plant extracts from Labiatae (Lamiaceae) growing naturally around Sirnak-Silopi, Turkey," International Journal of Pharmaceutical and Applied Sciences, vol. 1, no. 1, pp. 4-47, 2010.

[33] K. Bayoub, T. Baibai, D. Mountassif, A. Retmane, and A. Soukri, "Antibacterial activities of the crude ethanol extracts of medicinal plants against Listeria monocytogenes and some other pathogenic strains," African Journal of Biotechnology, vol. 9, no. 27, pp. 4251-4258, 2010.

[34] R. P. Samy, P. Gopalakrishnakone, M. Sarumathi, P. Houghton, and S. Ignacimuthu, "Purification of antibacterial agents from Tragia involucrata-a popular tribal medicine for wound healing," Journal of Ethnopharmacology, vol. 107, no. 1, pp. 99-106, 2006.

[35] K. M. Matthew, The Flora of Tamil Nadu Carnatic, Part I-III printed at the Diocesan Press, Madras, India, 1981-1983.

[36] A. W. Bauer, W. M. Kirby, J. C. Sherris, and M. Turck, "Antibiotic susceptibility testing by a standardized single disk method," American Journal of Clinical Pathology, vol. 45, no. 4, pp. 493496, 1966.

[37] National Committee for Clinical Laboratory Standards, Performance Standards For Antimicrobial Susceptibility Testing, 12th Informational Supplement, Approved standard M100-S12, National Committee for Clinical Laboratory Standards, Wayne, Pa, USA, 2002.

[38] E. A. Elsinghorst, "Measurement of invasion by gentamicin resistance," Methods in Enzymology, vol. 236, pp. 405-420, 1994.

[39] J. B. Harborne, Phytochemical Methods, Chapman and Hall, New York, NY, USA, 1976.

[40] H. Wagner, S. Bladt, and E. M. Zgairski, Plant Drug Analysis, Springer, New York, NY, USA, 1984.

[41] N. Myers, R. A. Mittermeler, C. G. Mittermeler, G. A. B. da Fonseca, and J. Kent, "Biodiversity hotspots for conservation priorities," Nature, vol. 403, no. 6772, pp. 853-858, 2000.

[42] R. Perumal Samy and S. Ignacimuthu, "Antibacterial activity of some folklore medicinal plants used by tribals in Western Ghats 
of India," Journal of Ethnopharmacology, vol. 69, no. 1, pp. 63-71, 2000.

[43] K. Nampoothiri, A. Chrispal, A. Begum, S. Jasmine, K. G. Gopinath, and A. Zachariah, "A clinical study of renal tubular dysfunction in Cleistanthus collinus (Oduvanthalai) poisoning," Clinical Toxicology, vol. 48, no. 3, pp. 193-197, 2010.

[44] M. Strathern, UsefulKnowledge, The Isiah Berlin Lecture. University of Manchester, Brunswick, UK.

[45] C. N. Sunil and A. K. Pradeep, "(2001) Another new species of Tripogon (Poaceae) from India,” Sida, vol. 19, pp. 803-806, 2005.

[46] S. Biber-Klemm and T. Cottier, Right To Plant Genetic Resources and Traditional Knowledge: Basic Issues and Prospective, Oxfordshire, Nosworthy Way, UK, 2006.

[47] V. S. Ramachandran, S. Shijo Joseph, and R. Aruna, "Ethnobotanical studies from amaravathy range of Indira Gandhi Wildlife Sanctuary, Western Ghats, Coimbatore District, Southern India," Ethnobotanical Leaflets, vol. 13, pp. 1069-1087, 2009.

[48] A. H. Rajasab and I. Mohamad, "Documentation of folk knowledge on edible wild plants of North Karnataka," Indian Journal of Traditional Knowledge, vol. 3, no. 4, pp. 419-429, 2004.

[49] M. Rajadurai, V. G. Vidhya, M. Ramya, and A. Bhaskar, "Ethnomedicinal plants used by the traditional healers of pachamalai hills, Tamilnadu, India," Studies on Ethno-Medicine, vol. 3, no. 1, pp. 39-41, 2009.

[50] S. K. Adesina, O. Idowu, A. O. Ogundaini et al., "Antimicrobial constituents of the leaves of Acalypha wilkesiana and Acalypha hispida," Phytotherapy Research, vol. 14, no. 5, pp. 371-374, 2000.

[51] M. T. Gutierrez-Lugo, M. P. Singh, W. M. Maiese, and B. N. Timmermann, "New antimicrobial cycloartane triterpenes from Acalypha communis," Journal of Natural Products, vol. 65, no. 6, pp. 872-875, 2002.

[52] A. K. Tyagi and A. Malik, "Antimicrobial potential and chemical composition of Mentha piperita oil in liquid and vapour phase against food spoiling microorganisms," Food Control, vol. 22, no. 11, pp. 1707-1714, 2011.

[53] G. Devendran and U. Balasubramanian, "Qualitative phytochemical screening and GC-MS analysis of Ocimum sanctum L. leaves," Asian Journal of Plant Science and Research, vol. 1, no. 4, pp. 44-48, 2011.

[54] V. C. Jain, N. M. Patel, D. P. Shah, P. K. Patel, and B. H. Joshi, "Antioxidant and antimicrobial activities of Alangium salvifolium (L.f.) Wang root," Global Journal of Pharmacology, vol. 4, no. 1, pp. 13-18, 2010.

[55] P. Swapna Latha and K. Kannabiran, "Antimicrobial activity and phytochemicals of Solanum trilobatum Linn," African Journal of Biotechnology, vol. 5, no. 23, pp. 2402-2404, 2006.

[56] M. Ushal, S. Ragini, and S. M. A. Naqvi, "Antibacterial activity of acetone and ethanol extracts of Cinnamon (Cinnamomum zeylanicum) and Ajowan (Trachyspermum ammi) on four food spoilage bacteria," International Research Journal of Biological Sciences, vol. 1, no. 4, pp. 7-11, 2012.

[57] S. Prabuseenivasan, M. Jayakumar, and S. Ignacimuthu, "In vitro antibacterial activity of some plant essential oils," $B M C$ Complementary and Alternative Medicine, vol. 6, article 39, pp. $1-8,2006$.

[58] G. A. Ayoola, F. M. Lawore, T. Adelowotan et al., "Chemical analysis and antimicrobial activity of the essential oil of Syzigium aromaticum (clove)," African Journal of Biotechnology, vol. 7, no. 13, pp. 2227-2231, 2008.
[59] Y. J. Fu, L. Chen, Y. Zu et al., “The antibacterial activity of clove essential oil against Propionibacterium acnes and its mechanism of action," Archives of Dermatology, vol. 145, no. 1, pp. 86-88, 2009.

[60] D. Venkatesan, C. M. Karrunakarn, S. Selva Kumar, and P. T. Palani Swamy, "Identification of phytochemical constituents of Aegle marmelos responsible for antimicrobial activity against selected pathogenic organisms," Ethnobotanical Leaflets, vol. 13, pp. 1362-1372, 2009.

[61] P. Arun, K. G. Purushotham, J. Johnsy jayarani, and V. Kumari, "In vitro Antibacterial Activity of Oldenlandia umbellata an Indian medicinal Plant," Journal of Pharmaceutical Science and Technology, vol. 2, no. 4, pp. 198-201, 2010.

[62] A. Manjamalai, R. Sardar Sathyajith Singh, C. Guruvayoorappan, and V. M. Berlin Grace, "Analysis of phytochemical constituents and antimicrobial activity of some medicinal plants in Taminadu, India," Global Journal of Biotechnology and Biochemistry, vol. 5, no. 2, pp. 120-128, 2010.

[63] E. Haslam, "Natural polyphenols (vegetable tannins) as drugs: Possible modes of action," Journal of Natural Products, vol. 59, no. 2, pp. 205-215, 1996.

[64] Y. Cai, Q. Luo, M. Sun, and H. Corke, "Antioxidant activity and phenolic compounds of 112 traditional Chinese medicinal plants associated with anticancer," Life Sciences, vol. 74, no. 17, pp. 2157-2184, 2004.

[65] B. Shan, Y. Z. Cai, M. Sun, and H. Corke, "Antioxidant capacity of 26 spice extracts and characterization of their phenolic constituents," Journal of Agricultural and Food Chemistry, vol. 53, no. 20, pp. 7749-7759, 2005.

[66] R. J. W. Lambert, P. N. Skandamis, P. J. Coote, and G.-J. E. Nychas, "A study of the minimum inhibitory concentration and mode of action of oregano essential oil, thymol and carvacrol," Journal of Applied Microbiology, vol. 91, no. 3, pp. 453-462, 2001.

[67] M. Raccach, "The antimicrobial activity of phenolic antioxidants in food: a review," Journal of Food Safety, vol. 6, no. 3, pp. 141-170, 1984.

[68] M. Taniguchi, Y. Yano, E. Tada et al., "Mode of action of polygodial, an antifungal sesquiterpene dialdehyde," Agricultural and Biological Chemistry, vol. 52, no. 6, pp. 1409-1414, 1988.

[69] G. J. E. Nychas, "Natural antimicrobials from plants," in New Methods of Food Preservation, G. W. Gould, Ed., pp. 58-89, Blackie Academic, London, UK, 1995.

[70] S. Burt, "Essential oils: their antibacterial properties and potential applications in foods-a review," International Journal of Food Microbiology, vol. 94, no. 3, pp. 223-253, 2004. 


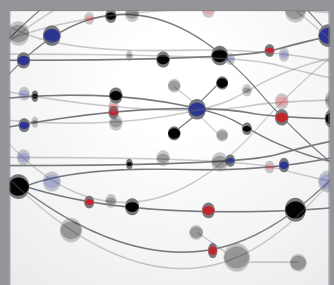

The Scientific World Journal
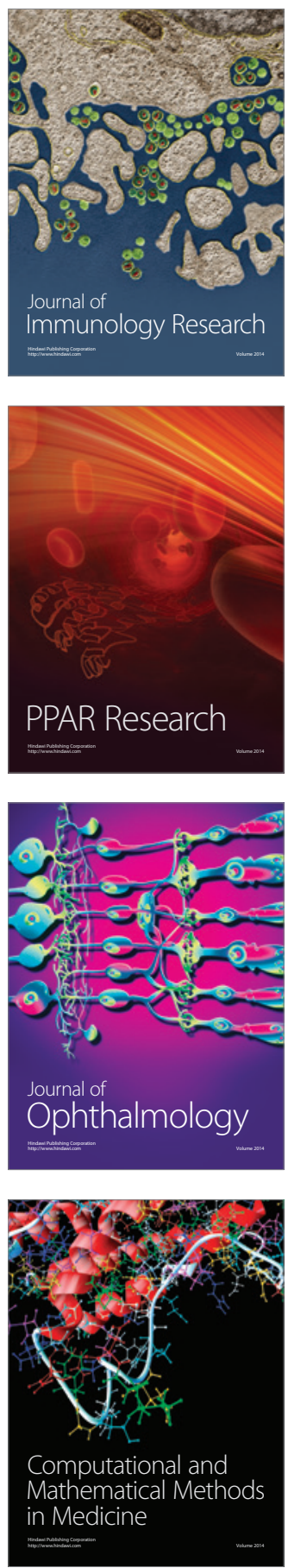

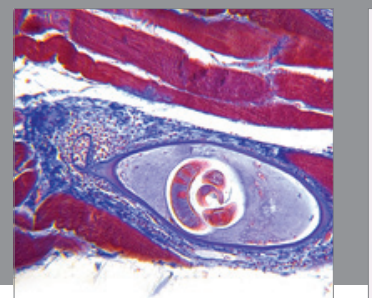

Gastroenterology

Research and Practice
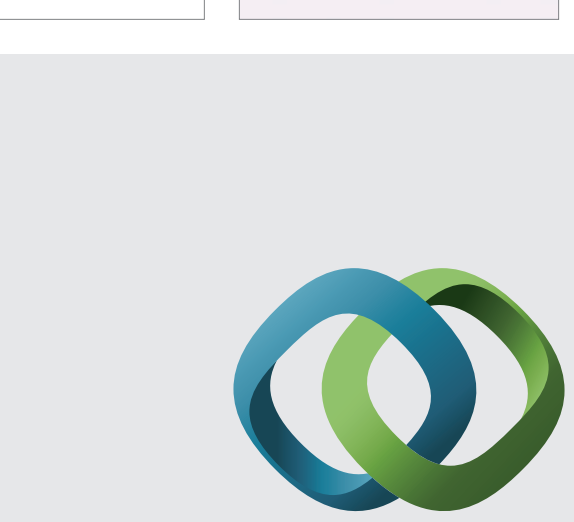

\section{Hindawi}

Submit your manuscripts at

http://www.hindawi.com
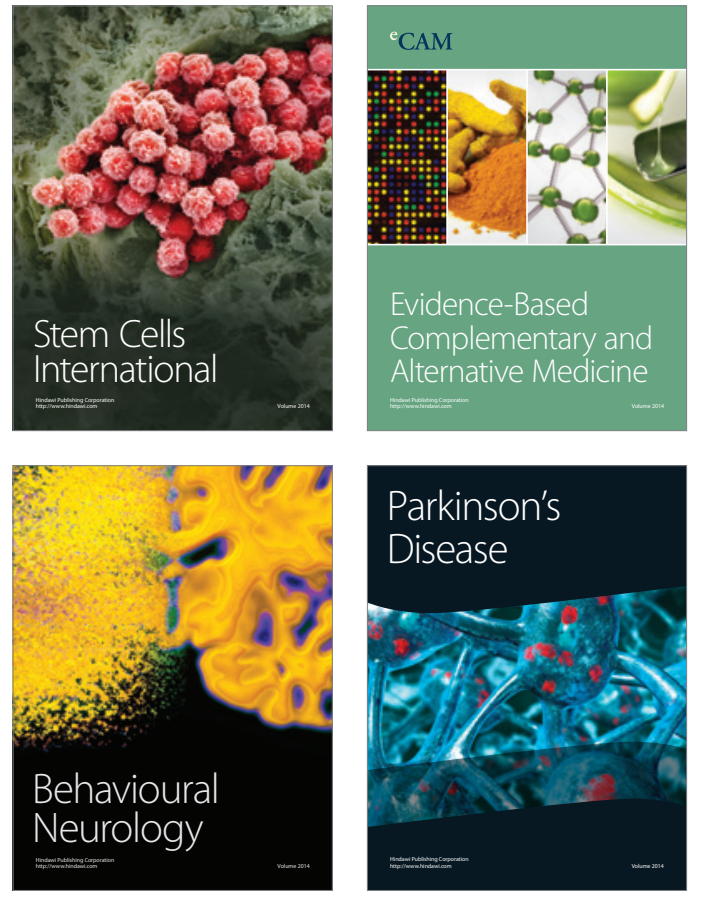
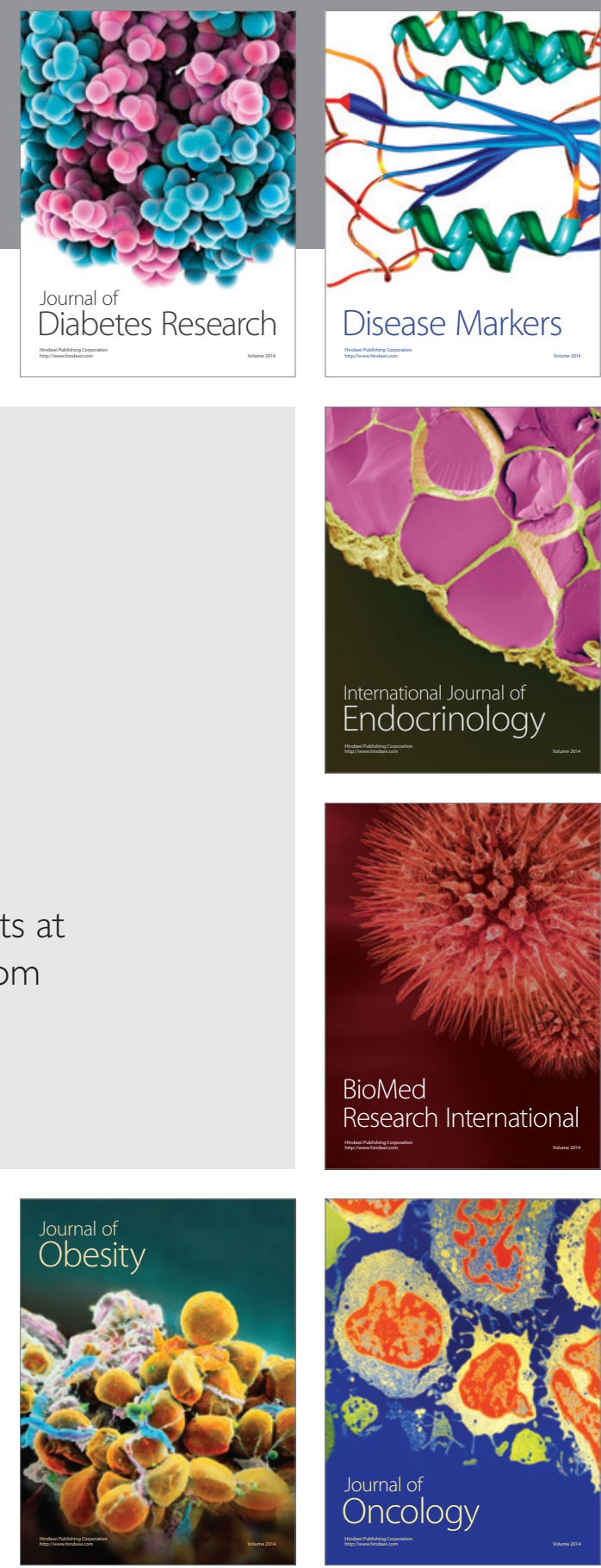

Disease Markers
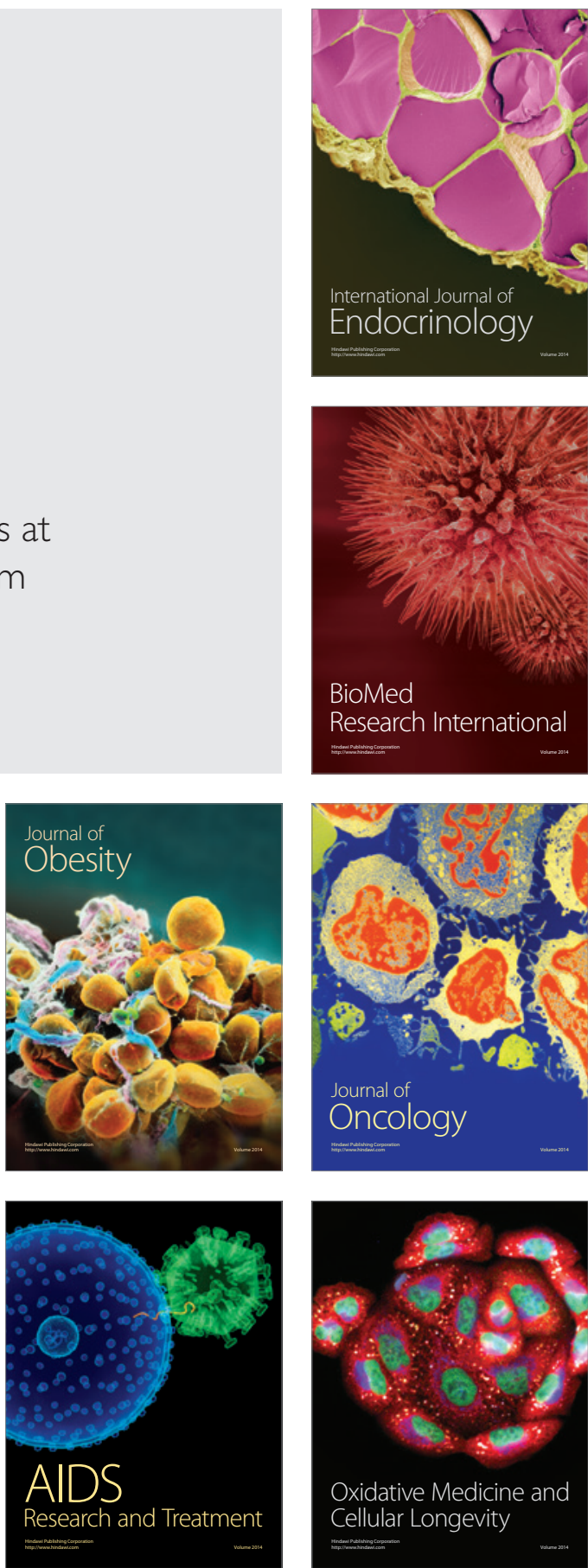